\title{
Factors affecting conformation in proline-containing peptides
}

\author{
Carol M. Taylor, ${ }^{\dagger \dagger}$ Renaud Hardré, ${ }^{\dagger}$ Patrick J. B. Edwards, ${ }^{\dagger}$ \\ Jae H. Park ${ }^{\ddagger}$ \\ Institute of Fundamental Sciences, Massey University, Private Bag 11-222, Palmerston \\ North, New Zealand, and Department of Chemistry, University of Auckland, Private \\ Bag 92019, Auckland, New Zealand \\ C.M.Taylor@massey.ac.nz. \\ Received Date (will be automatically inserted after manuscript is \\ accepted)
}

\section{SUPPORTING INFORMATION}

Procedures for the preparation of compounds 1, 2, 4, 5 and $6 ;{ }^{1} \mathrm{H}$ and ${ }^{13} \mathrm{C}$ NMR spectra for all intermediates and final products and tables of NMR and thermodynamic data.

\section{Experimental Procedures}

\section{General}

Synthesis of dipeptides: reagents were purchased and purified as described previously. ${ }^{1}$

Purification of dipeptides: this was performed by RP-HPLC using $250 \mathrm{~mm}$ long C18 columns $(4.6 \mathrm{~mm}$ diameter Spherisorb S10 ODS2, $10 \mathrm{~mm}$ diameter Econosil, $22 \mathrm{~mm}$ diameter Econosil), eluting with flow rates of $0.6,2.8$ and $12 \mathrm{~mL} \mathrm{m^{-1 }}$ respectively. The following gradient of acetonitile in water was employed: $20-30 \%$ over 8 min; $30-95 \%$ over $2 \mathrm{~min}$; $95 \%$ for $5 \mathrm{~min}$; $95-20 \%$ over 5 min. The eluent was monitored at $218 \mathrm{~nm}$ and $259 \mathrm{~nm}$; the compounds absorbed approximately 10-fold less strongly at $259 \mathrm{~nm}$ than they did at $218 \mathrm{~nm}$. Relevant fractions of eluent were concentrated by lyophilization.

\footnotetext{
${ }^{1}$ (a) Weir, C. A.; Taylor, C. M. J. Org. Chem. 1999, 64, 1554-1558; (b) Taylor, C. M.; Barker, W. D.; Weir, C. A.; Park, J. H. J. Org. Chem. 2002, 67, 4466-4474.
} 
S 2

NMR studies. Samples for NMR experiments were prepared by dissolving 2-3 mg of compound in $\mathrm{D}_{2} \mathrm{O}$ $(0.5 \mathrm{~mL})$. The $\mathrm{pH}$ value, without correction for isotope effects, was 7.56. The spectra were recorded on a Bruker AVANCE 400 spectrometer. Assignments have been given only for compounds where these have been determined unequivocally using COSY, HMQC, HMBC and NOESY experiments. In the many cases where a spectrum reflects the presence of two species arising from trans and cis rotamers about the X-Pro amide bond, the following conventions have been adopted for reporting. For ${ }^{1} \mathrm{H}$ spectra: chemical shift (multiplicity, \#protons ${ }^{\text {trans }} / \#$ protons ${ }^{c i s}$, assignment/s). For ${ }^{13} \mathrm{C}$ spectra: where signals are clearly part of a pair they are listed, X.X (X.X), where the first signal refers to the trans species and the second, in parentheses, to the cis. ${ }^{1} \mathrm{H}$ spectra were referenced relative to residual HOD at $4.65 \mathrm{ppm} .{ }^{13} \mathrm{C}$ spectra were referenced relative to external DSS (2,2-dimethyl-2-silapentane-5-sulfonate, sodium salt). The ratio of trans:cis rotamers was determined by integration of all well-resolved peaks in the onedimensional ${ }^{1} \mathrm{H}$ NMR spectra, and averaging of these results. Variable temperature experiments were conducted from $25-80{ }^{\circ} \mathrm{C}$ with a $5^{\circ} \mathrm{C}$ interval.

Ac-Pro-OMe (1) - this compound is commercially available from Bachem and Advanced ChemTech, but was prepared by acetylation of proline methyl ester hydrochloride and purified by flash chromatography, eluting with $0-5 \% \mathrm{MeOH}$ in $\mathrm{CH}_{2} \mathrm{Cl}_{2} \cdot{ }^{1} \mathrm{H} \mathrm{NMR}\left(400 \mathrm{MHz}, \mathrm{D}_{2} \mathrm{O}, K_{t / c}=5.4\right.$ at $\left.298 \mathrm{~K}\right) \delta$ 1.65-1.82 (m, $1 \mathrm{H}^{c i s}$, Pro $\left.\gamma^{\text {'is }}\right), 1.85-1.95\left(\mathrm{~m}, 3 \mathrm{H}^{\text {trans }} / 1 \mathrm{H}^{\text {cis }}\right.$, Pro $\beta^{{ }^{\text {trans }}}$, Pro $\gamma^{\text {trans }}$, Pro $\gamma^{\text {trans }}$, Pro $\left.\gamma^{\text {cis }}\right), 1.88$ $\left(\mathrm{s}, 3 \mathrm{H}^{c i s}, \mathrm{Ac}^{c i s}\right), 2.00\left(\mathrm{~s}, 3 \mathrm{H}^{\text {trans }}, \mathrm{Ac}^{\text {trans }}\right), 2.06-2.30\left(\mathrm{~m}, 1 \mathrm{H}^{\text {trans }} / 2 \mathrm{H}^{c i s}, \operatorname{Pro} \beta^{\text {trans }}\right.$, Pro $\beta^{{ }^{c i s}}$, Pro $\left.\beta^{\text {cis }}\right), 3.30-$ $3.46\left(\mathrm{~m}, 2 \mathrm{H}^{c i s}, \operatorname{Pro\delta }^{{ }^{c i s}} \operatorname{Pro\delta }^{c i s}\right), 3.46-3.59\left(\mathrm{~m}, 1 \mathrm{H}^{\text {trans }}\right.$, $\left.\operatorname{Pro\delta }^{\text {trans }}\right), 3.53\left(\mathrm{dd}, J=14.6,7.3 \mathrm{~Hz}, 1 \mathrm{H}^{\text {trans }}\right.$, $\left.\operatorname{Pro\delta }^{\text {trans }}\right), 3.64\left(\mathrm{~s}, 3 \mathrm{H}^{\text {trans }}, \mathrm{OMe}^{\text {trans }}\right), 3.68\left(\mathrm{~s}, 3 \mathrm{H}^{\text {cis }}, \mathrm{OMe}^{\text {cis }}\right), 4.32\left(\mathrm{dd}, \quad J=8.4,4.2 \mathrm{~Hz}, 1 \mathrm{H}^{\text {trans }}\right.$,

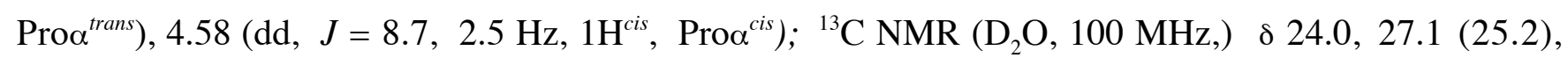
32.0 (33.5), 51.2 (49.4), 55.6 (56.0), 61.8 (63.4), 175.5 (175.9), 177.7 (177.2).

\section{Ac-Pro-NHMe (2)}

I. Boc-Pro-NHMe. Boc $_{2} \mathrm{O}$ (948 mg, $4.34 \mathrm{mmol}, 2$ equiv.) was added to a solution of $L$-proline (250 mg, 2.17 mmol, 1.0 equiv.) and triethylamine (303 $\mu \mathrm{L} 2.17 \mathrm{mmol}, 1.0$ equiv.) in dry methanol (6 $\mathrm{mL})$. The solution was heated at reflux for $3 \mathrm{~h}$ and then concentrated. The mixture was diluted with ethyl 


\section{S 3}

acetate $(50 \mathrm{~mL})$ and washed with ice-cold $1 \mathrm{M}$ aq. $\mathrm{HCl}(8 \mathrm{~mL})$. The aqueous layer was extracted with ethyl acetate $(4 \times 50 \mathrm{~mL})$. The organic layers were combined and dried $\left(\mathrm{MgSO}_{4}\right)$, filtered and concentrated. The residue was dissolved in dichloromethane $(5 \mathrm{~mL})$ and methylamine hydrochloride $(147$ mg, 2.17 mmol, 1.0 equiv.), BOP reagent (960 mg, $2.17 \mathrm{mmol}, 1.0$ equiv.) and triethylamine (760 $\mu \mathrm{L}$, $5.42 \mathrm{mmol}, 2.5$ equiv.) were added. The mixture was stirred at room temperature for $16 \mathrm{~h}$. The mixture was evaporated and the residue dissolved in $\mathrm{CH}_{2} \mathrm{Cl}_{2}(100 \mathrm{~mL})$, washed with $1 \mathrm{M}$ aq. $\mathrm{HCl}(5 \mathrm{~mL})$, sat'd aq. $\mathrm{NaHCO}_{3}(10 \mathrm{~mL})$ and brine $(10 \mathrm{~mL})$. The last two aqueous solutions were extracted with further dichloromethane $(2 \times 70 \mathrm{~mL})$. The organic layers were combined, dried $\left(\mathrm{MgSO}_{4}\right)$, filtered and concentrated. The residue was purified by flash column chromatography eluting with ethyl acetatehexanes (8:1). Relevant fractions were combined to give Boc-Pro-NHMe (407 mg, $82 \%) . \quad R_{f}=0.38$ (EtOAc); ${ }^{1} \mathrm{H}$ NMR (CD $\left.\mathrm{OD}, 400 \mathrm{MHz}\right) \delta 1.45$ (s, 9H $\left.{ }^{\text {trans }}\right), 1.50\left(\mathrm{~s}, 9 \mathrm{H}^{\text {cis }}\right), 1.81-2.01\left(\mathrm{~m}, 3 \mathrm{H}^{\text {trans }} / 3 \mathrm{H}^{\text {cis }}\right)$, 2.13-2.32 (m, $\left.1 \mathrm{H}^{\text {trans }} / 1 \mathrm{H}^{\text {cis }}\right), 2.78\left(\mathrm{~s}, 3 \mathrm{H}^{\text {trans }} / 3 \mathrm{H}^{\text {cis }}\right), 3.38-3.49\left(\mathrm{~m}, 1 \mathrm{H}^{\text {trans }} / 1 \mathrm{H}^{\text {cis }}\right), 3.49-3.58(\mathrm{~m}$, $\left.1 \mathrm{H}^{\text {trans }} / 1 \mathrm{H}^{\text {cis }}\right), 4.14\left(\mathrm{dd}, J=7.9,3.8 \mathrm{~Hz}, 1 \mathrm{H}^{\text {trans }}\right), 4.19$ (br. d, $\left.J=8.2 \mathrm{~Hz}, 1 \mathrm{H}^{\text {cis }}\right) ;{ }^{13} \mathrm{C}$ NMR $\left(\mathrm{CD}_{3} \mathrm{OD}\right.$, $100 \mathrm{MHz}$ ) $\delta 24.7$ (25.3), 26.3 (26.3), 28.6 (28.7), 32.4 (31.5), 47.8 (48.3), 62.0 (61.7), 81.3 (79.5), 155.9 (156.4), 176.3 (175.9).

II. Ac-Pro-NHMe (2). Boc-Pro-NHMe $(57 \mathrm{mg}, 0.25 \mathrm{mmol})$ was dissolved in a 1:1 (v/v) solution of TFA and dichloromethane $(2 \mathrm{~mL})$. The solution was stirred at room temperature for $30 \mathrm{~min}$ and concentrated. The residue was dissolved in pyridine $(1 \mathrm{~mL})$ and acetic anhydride $(1 \mathrm{~mL})$. The solution was stirred at room temperature for $19 \mathrm{~h}$ and concentrated. The residue was purified by flash column chromatography, eluting with 0-10\% MeOH in ethyl acetate. Relevant fractions were combined to give Ac-Pro-NHMe (2) (35 mg, 83\%). ${ }^{1} \mathrm{H}$ NMR (400 MHz, $\mathrm{D}_{2} \mathrm{O}, K_{t / c}=2.6$ at 298K) $\delta 1.64-2.26(\mathrm{~m}$, $4 \mathrm{H}^{\text {trans }} / 4 \mathrm{H}^{c i s}, \operatorname{Pro} \beta^{\text {trans }}, \operatorname{Pro} \beta^{\text {trans }}$, Pro $\left.{ }^{{ }^{t r a n s}}, \operatorname{Pro} \gamma^{\text {trans }}, \operatorname{Pro} \beta^{{ }^{\prime c i s}}, \operatorname{Pro} \beta^{c i s}, \operatorname{Pro} \gamma^{{ }^{c i s}}, \operatorname{Pro} \gamma^{c i s}\right), 1.82\left(\mathrm{~s}, 3 \mathrm{H}^{c i s}\right.$, $\left.\mathrm{Ac}^{\text {cis }}\right), 1.96\left(\mathrm{~s}, 3 \mathrm{H}^{\text {trans }}, \mathrm{Ac}^{\text {trans }}\right), 2.57\left(\mathrm{~s}, 3 \mathrm{H}^{\text {trans }}, \mathrm{NHMe}^{\text {trans }}\right), 2.62\left(\mathrm{~s}, 3 \mathrm{H}^{\text {cis }}, \mathrm{NHMe}^{\text {cis }}\right), 3.25-3.59(\mathrm{~m}$,

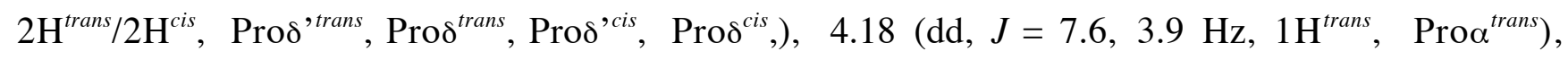

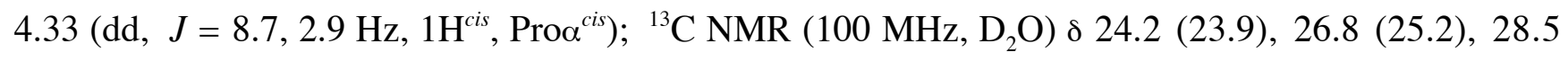
(28.7), 32.7 (34.3), 51.4 (49.8), 63.1 (64.6), 176.1 (176.3), 177.7 (177.6).

\section{Ac-Gly-Pro-NHMe (4).}


I. Boc-Gly-Pro-NHMe. BOP reagent (650 mg, $1.47 \mathrm{mmol}, 1.0$ equiv.) was added to a solution of Boc-Gly-Pro-OH (400 mg, 1.47 mmol, 1.0 equiv.), methylamine hydrochloride (99 mg, 1.47 mmol, 1.0 equiv.) and triethylamine (1.3 mL, $9.23 \mathrm{mmol}, 3.0$ equiv.) in acetonitrile $(15 \mathrm{~mL})$. The solution was stirred at room temperature for $18 \mathrm{~h}$ and then concentrated. The mixture was diluted with ethyl acetate (75 $\mathrm{mL})$, washed with $1 \mathrm{M}$ aq. $\mathrm{HCl}(40 \mathrm{~mL})$, sat'd aq. $\mathrm{NaHCO}_{3 .}(40 \mathrm{~mL})$, water $(20 \mathrm{~mL})$ and brine $(40 \mathrm{~mL})$. The organic layer was dried $\left(\mathrm{MgSO}_{4}\right)$, filtered and concentrated. The residue was purified by flash column chromatography, eluting with $0-10 \% \mathrm{MeOH}$ in ethyl acetate. After evaporation of the relevant fractions, Boc-Gly-Pro-NHMe was obtained (217 mg, $52 \%) . \quad R_{f}=0.37$ (EtOAc-MeOH 9:1). ${ }^{1} \mathrm{H}$ NMR $\left(\mathrm{CDCl}_{3}\right.$, $400 \mathrm{MHz}) \delta 1.45$ (s, 9H), 1.74-2.34 (m, 4H), 2.70 (d, $J=4.8 \mathrm{~Hz}, 3 \mathrm{H}), 3.37$ (dd, $J=16.2,8.1 \mathrm{~Hz}$, $1 \mathrm{H}), 3.47-3.60(\mathrm{~m}, 1 \mathrm{H}), 3.88(\mathrm{~d}, J=4.6 \mathrm{~Hz}, 2 \mathrm{H}), 4.48(\mathrm{dd}, J=7.7,2.2 \mathrm{~Hz}, 1 \mathrm{H}), 5.50(\mathrm{~d}, J=4.8$ $\mathrm{Hz}, 1 \mathrm{H}), 6.92(\mathrm{~d}, J=4.6 \mathrm{~Hz}, 1 \mathrm{H}) ;{ }^{13} \mathrm{C} \mathrm{NMR}\left(\mathrm{CDCl}_{3}, 100 \mathrm{MHz}\right) \delta 24.8,26.2,27.9,28.3,43.1,46.4$, 60.2, 79.8, 155.9, 168.7, 171.4; HRMS $\left(\mathrm{FAB}^{+}, \mathrm{NBA}, \mathrm{MeOH}\right)$ calcd for $(\mathrm{MH})^{+} \mathrm{C}_{13} \mathrm{H}_{23} \mathrm{~N}_{3} \mathrm{O}_{4}$ : 286.1759; obsd 286.1767 .

II. Ac-Gly-Pro-NHMe (4). Boc-Gly-Pro-NHMe (100 mg, $0.35 \mathrm{mmol})$ was dissolved in a 1:1 (v/v) solution of TFA and dichloromethane $(6 \mathrm{~mL})$. The solution was stirred at room temperature for $30 \mathrm{~min}$ and concentrated. The residue was dissolved in pyridine $(1 \mathrm{~mL})$ and acetic anhydride $(1 \mathrm{~mL})$. The solution was stirred at room temperature for $19 \mathrm{~h}$ and concentrated. The residue was purified by flash column chromatography, eluting with $0-20 \% \mathrm{MeOH}$ in ethyl acetate. Relevant fractions were combined to give Ac-Gly-Pro-NHMe (4) (35 mg, 44\%). ${ }^{1} \mathrm{H}$ NMR (400 MHz, $\mathrm{D}_{2} \mathrm{O}, K_{t / c}=5.5$ at 298K) $\delta 1.67-1.77(\mathrm{~m}$, $1 \mathrm{H}^{c i s}$, Pro $\left.\gamma^{\prime c i s}\right), 1.77-1.96\left(\mathrm{~m}, 3 \mathrm{H}^{\text {trans }} / 1 \mathrm{H}^{\text {cis }}\right.$, Pro $\beta^{\text {trans }}$, Pro ${ }^{\text {trans }}$, Pro $\gamma^{\text {trans }}$, Pro $\left.\gamma^{c i s}\right), 1.92\left(\mathrm{~s}, 3 \mathrm{H}^{\text {cis }}\right.$, $\left.A c^{\text {cis }}\right), 1.93\left(\mathrm{~s}, 3 \mathrm{H}^{\text {trans }}, \mathrm{Ac}^{\text {trans }}\right), 1.96-2.04\left(\mathrm{~m}, 1 \mathrm{H}^{\text {cis }}\right.$, Proß $\left.{ }^{\text {cis }}\right), 2.06-2.15\left(\mathrm{~m}, 1 \mathrm{H}^{\text {trans }}\right.$, Pro $\left.{ }^{\text {trans }}\right), 2.17-$ $2.26\left(\mathrm{~m}, 1 \mathrm{H}^{\text {cis }}, \operatorname{Pro}{ }^{c i s}\right), 2.61\left(\mathrm{~s}, 3 \mathrm{H}^{\text {trans }}\right.$, $\left.\mathrm{NHMe}^{\text {trans }}\right), 2.66\left(\mathrm{~s}, 3 \mathrm{H}^{\text {cis }}, \mathrm{NHMe}^{c i s}\right), 3.35-3.58(\mathrm{~m}$,

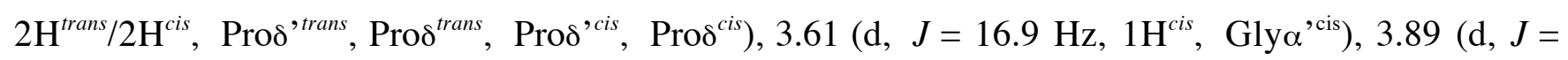

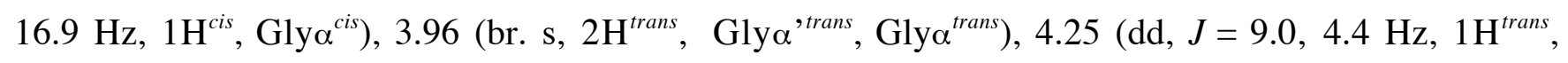
Proc $\left.{ }^{\text {trans }}\right), 4.41\left(\mathrm{dd}, J=8.8,2.7 \mathrm{~Hz}, 1 \mathrm{H}^{c i s}\right.$, Pro $\left.{ }^{c i s}\right) ;{ }^{13} \mathrm{C}$ NMR $\left(100 \mathrm{MHz}, \mathrm{D}_{2} \mathrm{O}\right) \mathrm{d} 24.3,27.0$ (24.6), 28.6 (28.8), 32.2 (34.5), 44.5 (44.3), 49.7 (50.3), 63.6 (63.2), 172.5 (172.8), 177.4, 177.5 (177.0, 177.3); HRMS (FAB ${ }^{+}$, glycerol, $\mathrm{MeOH}$ ) calcd for $(\mathrm{MH})^{+} \mathrm{C}_{10} \mathrm{H}_{18} \mathrm{~N}_{3} \mathrm{O}_{3}: 228.1348$; obsd 228.1348. 
Ac-Phe-Pro-OMe (5). Diphenylphosphoryl azide (150 $\mu \mathrm{L}, 160 \mathrm{mg}, 0.69 \mathrm{mmol}, 1.1$ equiv.) was added to a solution of $N$-acetylphenylalanine (143 mg, $0.69 \mathrm{mmol}, 1.1$ equiv.), proline methyl ester hydrochloride (102 mg, $0.62 \mathrm{mmol}, 1.0$ equiv.) and triethylamine (220 $\mu \mathrm{L}, 160 \mathrm{mg}, 1.57 \mathrm{mmol}, 2.5$ equiv.) in dry acetonitrile $(5 \mathrm{~mL})$. The solution was stirred at room temperature under nitrogen for $18 \mathrm{~h}$ and then concentrated. The residue was partitioned between ethyl acetate $(60 \mathrm{~mL})$ and water $(60 \mathrm{~mL})$. The organic layer was washed with brine $(60 \mathrm{~mL})$, dried over $\mathrm{MgSO}_{4}$, filtered and concentrated. The product was isolated from the residue by RP-HPLC to give Ac-Phe-Pro-OMe (5) (15 mg, 28\%). $R_{f} 0.33$ (95:5 $\left.\mathrm{CH}_{2} \mathrm{Cl}_{2}-\mathrm{MeOH}\right) ;{ }^{1} \mathrm{H} \mathrm{NMR}\left(400 \mathrm{MHz}, \mathrm{D}_{2} \mathrm{O}, K_{t / c}=4.20\right.$ at $\left.298 \mathrm{~K}\right) \delta 1.46-1.65\left(\mathrm{~m}, 4 \mathrm{H}^{c i s}\right.$, Pro $\beta^{{ }^{c i s}}$,

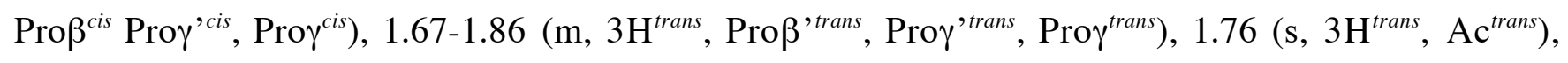
$1.78\left(\mathrm{~s}, 3 \mathrm{H}^{\text {cis }}, \mathrm{Ac}^{\text {cis }}\right), 2.02-2.16\left(\mathrm{~m}, 1 \mathrm{H}^{\text {trans }}, \operatorname{Pro} \beta^{\text {trans }}\right), 2.78\left(\mathrm{dd}, J=13.9,8.2 \mathrm{~Hz}, 1 \mathrm{H}^{\text {trans }}\right.$, Phe $\left.\beta^{\text {trans }}\right)$, $2.76\left(\mathrm{~m}, 2 \mathrm{H}^{c i s}\right.$, Phe $\beta^{c i s}$, Phe $\left.\beta^{c i s}\right), 2.96\left(\mathrm{dd}, J=13.9,6.5 \mathrm{~Hz}, 1 \mathrm{H}^{\text {trans }}\right.$, Phe $\left.\beta^{\text {trans }}\right), 3.10-3.27\left(\mathrm{~m}, 1 \mathrm{H}^{\text {trans }}\right.$, $\left.1 \mathrm{H}^{\text {cis }}, \operatorname{Pro\delta }^{\text {trans }}, \operatorname{Pro\delta }^{\text {cis }}\right), 3.35\left(\mathrm{q}, J=5.9 \mathrm{~Hz}, 1 \mathrm{H}^{c i s}, \operatorname{Pro\delta }^{c i s}\right), 3.57\left(\mathrm{~s}, 3 \mathrm{H}^{c i s}, \mathrm{OMe}^{\text {cis }}\right), 3.60\left(\mathrm{~s}, 3 \mathrm{H}^{\text {trans }}\right.$, $\left.\mathrm{OMe}^{\text {trans }}\right), 3.52-3.66\left(\mathrm{~m}, 1 \mathrm{H}^{\text {trans }}\right.$, $\left.\operatorname{Pro\delta }^{\text {trans }}\right), 3.84$ (br. d, $J=7.6 \mathrm{~Hz}, 1 \mathrm{H}^{c i s}$, Proc $\left.{ }^{\text {cis }}\right), 4.28(\mathrm{dd}, J=8.2$,

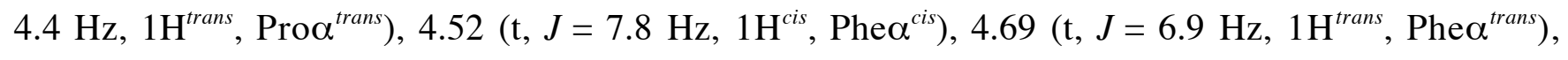

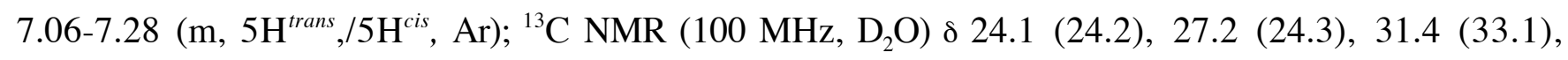
39.2 (41.0), 50.4 (49.4), 55.6 (55.5), 55.7 (55.9), 62.4 (62.3), 129.9 (130.2), 131.4, 132.1, (131.6, 132.0), 138.9 (138.9), 174.6 (174.5), 176.5, 177.2 (175.7, 176.7); HRMS (EI ${ }^{+}$) calcd for $\mathrm{C}_{17} \mathrm{H}_{22} \mathrm{O}_{4} \mathrm{~N}_{2}$ $\left(\mathrm{M}^{+}\right)$: 318.15796; obsd 318.15789.

\section{Ac-Phe-Pro-NHMe (6).}

I. Fmoc-Phe-Pro-NHMe. N-Hydroxysuccinimide (150 mg, $1.29 \mathrm{mmol}, 1.0$ equiv.), followed by DCC (265 mg, $1.29 \mathrm{mmol}, 1.0$ equiv.) was added to a solution of Fmoc-Phe-OH (500 mg, $1.29 \mathrm{mmol}$, 1.0 equiv. $)$ in $\mathrm{CH}_{2} \mathrm{Cl}_{2}(15 \mathrm{~mL})$ at $0{ }^{\circ} \mathrm{C}$ under $\mathrm{N}_{2}$. The solution was stirred at $0{ }^{\circ} \mathrm{C}$ for 20 min and then warmed to room temperature and stirred for a further $4 \mathrm{~h}$. The suspension was filtered through a plug of cotton in a Pasteur pipette. The filtrate was concentrated to $4 \mathrm{~mL}$ and then refrigerated for $2 \mathrm{~h}$. The suspension was filtered again and the residue evaporated to give a colorless foam which was dissolved in DMF (7.5 mL) and cooled to $0{ }^{\circ} \mathrm{C}$ under $\mathrm{N}_{2}$. L-Proline (150 mg, $1.29 \mathrm{mmol}, 1.0$ equiv.) was added as a 
solid in one portion, followed by the dropwise addition of diisopropylethylamine ( $225 \mu \mathrm{L}, 165 \mathrm{mg}, 1.29$ mmol, 1.0 equiv.). The solution was gradually warmed to room temperature and left to stir overnight. The mixture was diluted with ethyl acetate $(70 \mathrm{~mL})$ and washed with $2 \mathrm{M} \mathrm{HCl}(70 \mathrm{~mL})$. The acidic aqueous layer was back-extracted with ethyl acetate $(70 \mathrm{~mL})$. The organic layers were combined, washed with water $(140 \mathrm{~mL})$ and brine $(140 \mathrm{~mL})$, filtered through $\mathrm{MgSO}_{4}$ and concentrated to give Fmoc-PhePro-OH. This residue was dissolved in $\mathrm{CH}_{2} \mathrm{Cl}_{2}(15 \mathrm{~mL})$ and cooled to $0{ }^{\circ} \mathrm{C}$ under $\mathrm{N}_{2}$. Methylamine hydrochloride ( $87 \mathrm{mg}, 1.29 \mathrm{mmol}, 1.0$ equiv.) was added, followed by triethylamine (430 $\mu \mathrm{L}, 313 \mathrm{mg}$, $3.09 \mathrm{mmol}, 2.4$ equiv.) and finally BOP reagent $(571 \mathrm{mg}, 1.29 \mathrm{mmol}, 1.0$ equiv). The mixture was stirred at room temperature for $16 \mathrm{~h}$ and then diluted with $\mathrm{CH}_{2} \mathrm{Cl}_{2}(100 \mathrm{~mL})$, washed with $2 \mathrm{M} \mathrm{HCl}(100$ $\mathrm{mL})$, water $(100 \mathrm{~mL})$ and brine $(100 \mathrm{~mL})$, filtered through $\mathrm{MgSO}_{4}$ and concentrated. The product was isolated by flash chromatography, eluting with $2 \% \mathrm{MeOH}$ in EtOAc to give Fmoc-Phe-Pro-NHMe (375 mg, 58\%). $R_{f} 0.73$ (9:1 $\left.\mathrm{CH}_{2} \mathrm{Cl}_{2}-\mathrm{MeOH}\right) ;{ }^{1} \mathrm{H}$ NMR (400 MHz, $\mathrm{CDCl}_{3}$ ) o 1.68-1.98 (m, 3H), 2.28-2.38 $(\mathrm{m}, 1 \mathrm{H}), 2.75(\mathrm{~d}, J=4.7 \mathrm{~Hz}, 3 \mathrm{H}), 2.98-3.08(\mathrm{~m}, 2 \mathrm{H}), 3.42-3.66(\mathrm{~m}, 2 \mathrm{H}), 4.20(\mathrm{t}, J=7.0 \mathrm{~Hz}, 1 \mathrm{H})$, $4.32(\mathrm{dd}, J=10.5,7.1 \mathrm{~Hz}, 1 \mathrm{H}), 4.42(\mathrm{dd}, J=10.5,7.5 \mathrm{~Hz}, 1 \mathrm{H}), 4.54(\mathrm{dd}, J=7.1,1.8 \mathrm{~Hz}, 1 \mathrm{H}), 4.76$ (dd, $J=14.5,7.6 \mathrm{~Hz}, 1 \mathrm{H}), 5.73(\mathrm{~d}, J=8.6 \mathrm{~Hz}, 1 \mathrm{H}), 6.44(\mathrm{br} \mathrm{s}, 1 \mathrm{H}), 7.18-7.33(\mathrm{~m}, 7 \mathrm{H}), 7.31(\mathrm{t}, J=$ $7.5 \mathrm{~Hz}, 2 \mathrm{H}), 7.58(\mathrm{~m}, 2 \mathrm{H}), 7.77(\mathrm{~d}, J=7.5 \mathrm{~Hz}, 2 \mathrm{H}){ }^{13} \mathrm{C} \mathrm{NMR}\left(100 \mathrm{MHz}, \mathrm{CDCl}_{3}\right) \delta$ 24.9, 26.2, 27.1, $39.1,47.0,47.4,53.5,59.9,67.0,120.0,125.0,125.1,127.0,127.2,127.7,128.5,129.3,135.6$, 141.2, 143.7, 155.6, 170.9, 171.4; HRMS (FAB, NBA, MeOH) calcd for $(\mathrm{MH})^{+} \mathrm{C}_{30} \mathrm{H}_{32} \mathrm{~N}_{3} \mathrm{O}_{4}$ : 498.2393; obsd 498.2389.

II. Ac-Phe-Pro-NHMe (6). Diethylamine (2 mL) was added to a solution of Fmoc-Phe-Pro-NHMe (115 mg, $0.230 \mathrm{mmol}, 1.0$ equiv.) in acetonitrile $(15 \mathrm{~mL})$. The solution was stirred at room temperature for $1 \mathrm{~h}$, concentrated and then concentrated twice more from acetonitrile. The residue was suspended in $\mathrm{CH}_{2} \mathrm{Cl}_{2}(10 \mathrm{~mL})$. Triethylamine (153 $\mu \mathrm{L}, 111 \mathrm{mg}, 2.0$ equiv.) was added, followed by acetyl chloride (41 $\mu 1,45 \mathrm{mg}, 1.05$ equiv.). The solution was stirred at room temperature for $16 \mathrm{~h}$, then evaporated. The residue was filtered through a plug of silica gel washing with ethyl acetate $(200 \mathrm{~mL})$, followed by a mixture of 9:1 EtOAc-methanol $(300 \mathrm{~mL})$. The latter solution was concentrated to give a colorless foam $\left(170 \mathrm{mg}\right.$ ) which was further purified by RP-HPLC to give Ac-Phe-Pro-NHMe (6) $(73 \mathrm{mg} ; 42 \%) . \quad R_{f}$ 
$0.69\left(95: 5 \mathrm{CH}_{2} \mathrm{Cl}_{2}-\mathrm{MeOH}\right) ;{ }^{1} \mathrm{H}$ NMR $\left(\mathrm{D}_{2} \mathrm{O}, 400 \mathrm{MHz}, K_{t / c}=2.10\right.$ at $\left.298 \mathrm{~K}\right) \delta 1.33-1.58\left(\mathrm{~m}, 3 \mathrm{H}^{c i s}\right.$, $\operatorname{Pro} \beta^{{ }^{c i s}}$, Pro ${ }^{{ }^{c i s}}$, Pro $\left.\gamma^{c i s}\right), 1.63-1.84\left(\mathrm{~m}, 3 \mathrm{H}^{\text {trans }} / 1 \mathrm{H}^{c i s}\right.$, Pro $\beta^{\text {trans }}$, Pro $\gamma^{\text {trans }}$, Pro $\gamma^{\text {trans }}$, Pro $\left.\beta^{c i s}\right), 1.80(\mathrm{~s}$, $\left.3 \mathrm{H}^{\text {trans }}, \mathrm{Ac}^{\text {trans }}\right), 1.85\left(\mathrm{~s}, 3 \mathrm{H}^{c i s}, \mathrm{Ac}^{c i s}\right), 1.99-2.12\left(\mathrm{~m}, 1 \mathrm{H}^{\text {trans }}\right.$, Pro $\left.\beta^{\text {trans }}\right), 2.57\left(\mathrm{~s}, 3 \mathrm{H}^{\text {cis }}, \mathrm{NHMe}^{c i s}\right), 2.59$ $\left(\mathrm{s}, 3 \mathrm{H}^{\text {trans }}, \mathrm{NHMe}^{\text {trans }}\right), 2.78\left(\mathrm{dd}, J=14.0,8.5 \mathrm{~Hz}, 1 \mathrm{H}^{\text {trans }}\right.$, Phe $\left.\beta^{\text {trans }}\right), 2.80(\mathrm{dd}, J=12.9,9.1 \mathrm{~Hz}$, $1 \mathrm{H}^{c i s}$, Phe $\left.\beta^{{ }^{c i s}}\right), 2.85\left(\mathrm{dd}, J=12.9,6.6 \mathrm{~Hz}, 1 \mathrm{H}^{c i s}, P h e \beta^{c i s}\right), 3.00\left(\mathrm{dd}, J=14.0,6.1 \mathrm{~Hz}, 1 \mathrm{H}^{\text {trans }}\right.$, Phe $\left.\beta^{\text {trans }}\right), 3.17\left(\mathrm{dt}, J=11.7,8.1 \mathrm{~Hz}, 1 \mathrm{H}^{c i s}\right.$, Prod $\left.{ }^{c i s}\right), 3.31-3.42\left(\mathrm{~m}, 1 \mathrm{H}^{c i s}\right.$, Prod $\left.\delta^{c i s}\right), 3.36(\mathrm{dd}, J=10.0$, $6.5 \mathrm{~Hz}, 1 \mathrm{H}^{\text {trans }}$, Prod $\left.{ }^{\text {trans }}\right), 3.47\left(\mathrm{~d}, J=6.0 \mathrm{~Hz}, 1 \mathrm{H}^{\text {cis }}\right.$, Proc $\left.{ }^{c i s}\right), 3.65\left(\mathrm{dt}, J=10.0,6.8 \mathrm{~Hz}, 1 \mathrm{H}^{\text {trans }}\right.$, $\left.\operatorname{Prod}^{\text {trans }}\right), 4.38\left(\mathrm{dd}, J=9.1,6.6 \mathrm{~Hz}, 1 \mathrm{H}^{c i s}\right.$, Pro $\left.{ }^{c i s}\right), 4.70\left(\mathrm{dd}, J=8.5,6.1 \mathrm{~Hz}, 1 \mathrm{H}^{\text {trans }}\right.$, Phe $\left.\alpha^{\text {trans }}\right), 5.20$ $\left(\mathrm{dd}, J=8.6,5.1 \mathrm{~Hz}, 1 \mathrm{H}^{\text {trans }}\right.$, Phe $\left.{ }^{\text {trans }}\right), 7.01-7.23(\mathrm{~m}, 5 \mathrm{H}, \mathrm{Ar}) ;{ }^{13} \mathrm{C} \mathrm{NMR}\left(100 \mathrm{MHz}, \mathrm{D}_{2} \mathrm{O}\right) \delta 24.2$ (24.3), 27.3 (24.4), 28.7 (28.8), 32.0 (33.8), 39.2 (41.0), 50.7 (49.8), 55.8 (55.8), 63.7 (63.5), 130.0 (130.3), 131.5, 132.0, (131.7, 132.0), 139.1 (138.3), 174.7 (174.9), 176.3, 177.1, (175.6, 176.2); HRMS (FAB $\left.{ }^{+}, \mathrm{NBA}, \mathrm{MeOH}\right)$ calcd for $(\mathrm{MH})^{+} \mathrm{C}_{17} \mathrm{H}_{24} \mathrm{O}_{3} \mathrm{~N}_{3}(\mathrm{MH})^{+}$: 318.1818; obsd 318.1811. 
${ }^{1} \mathrm{H}$ and ${ }^{13} \mathrm{C}$ NMR spectra

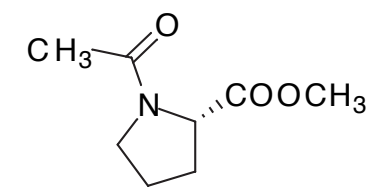

Compound 1

${ }^{1} \mathrm{H}$ NMR spectrum at $400 \mathrm{MHz}$ in $\mathrm{D}_{2} \mathrm{O}$

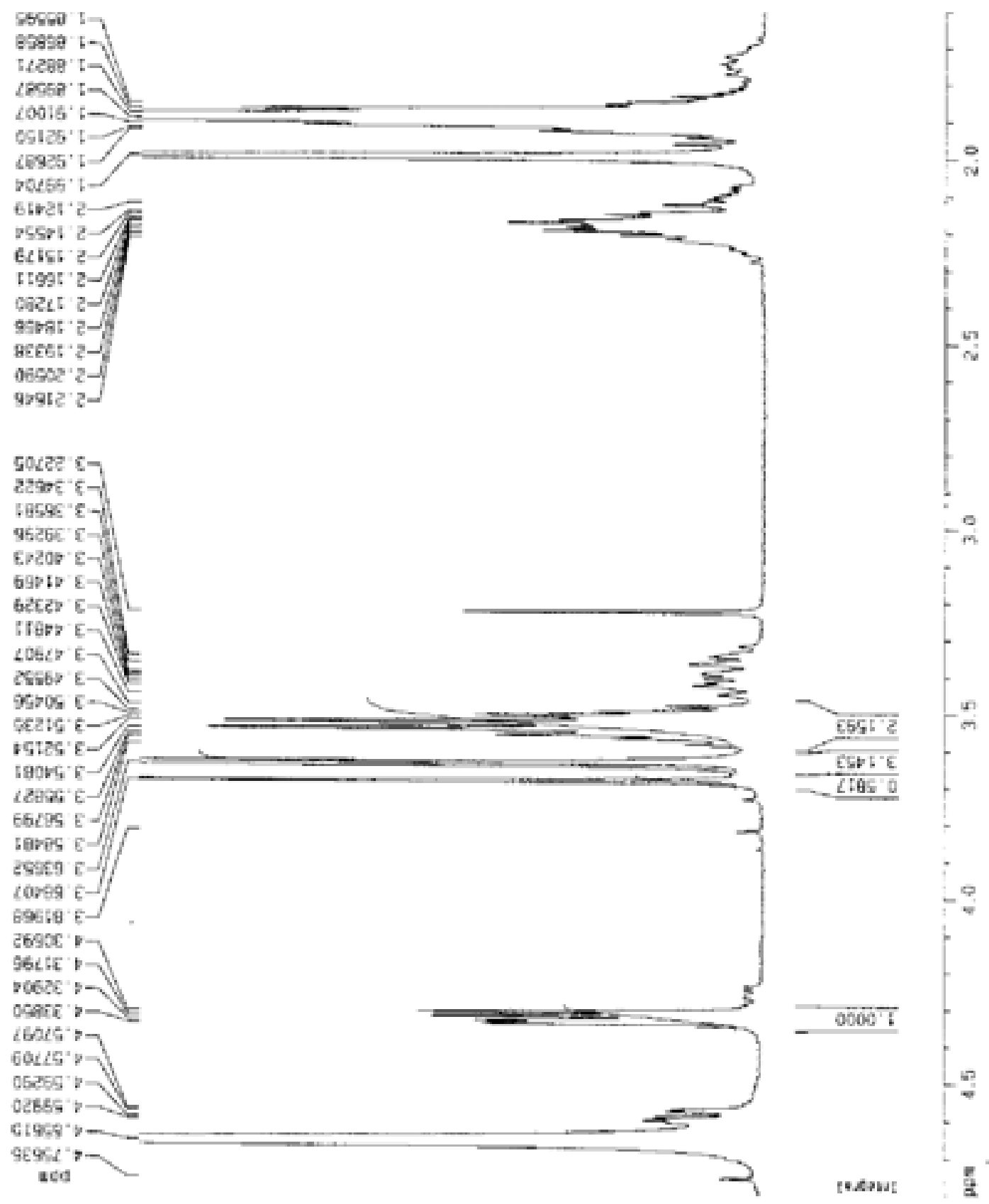


S 9

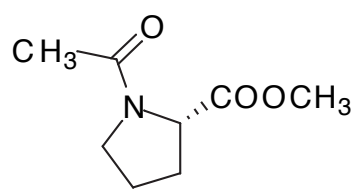

Compound 1

${ }^{13} \mathrm{C}$ NMR spectrum at $100 \mathrm{MHz}$ in $\mathrm{D}_{2} \mathrm{O}$

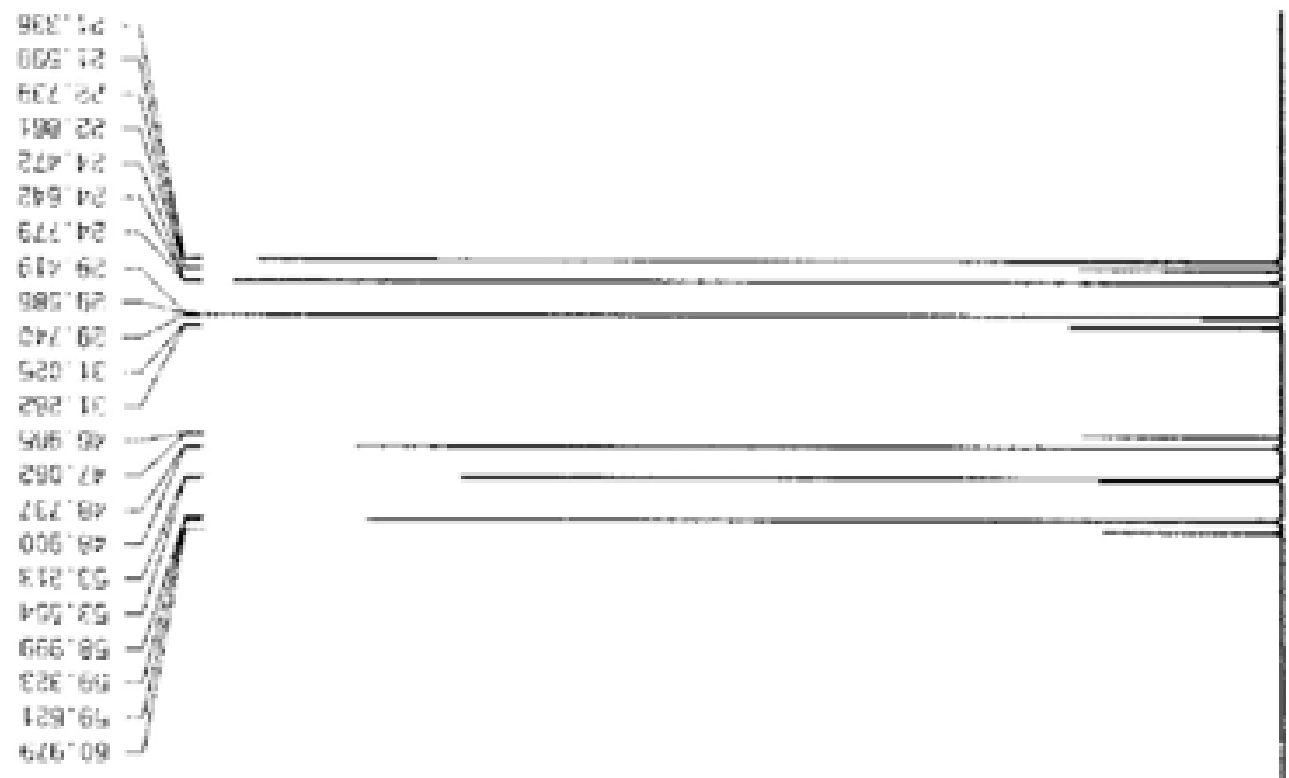

$560 \Sigma 2:$

证它:

bit $5: 2 \mathrm{t}-\mathrm{C}$

0 de' $72 \mathrm{t}-7 \mathrm{f}$

$D E C^{\prime} \mathrm{GL}=17$

Act 9.4 . 
$\mathrm{S} 10$

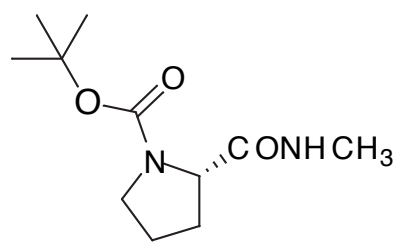

Boc-Pro-NHMe

${ }^{1} \mathrm{H}$ NMR spectrum at $400 \mathrm{MHz}$ in $\mathrm{CD}_{3} \mathrm{OD}$

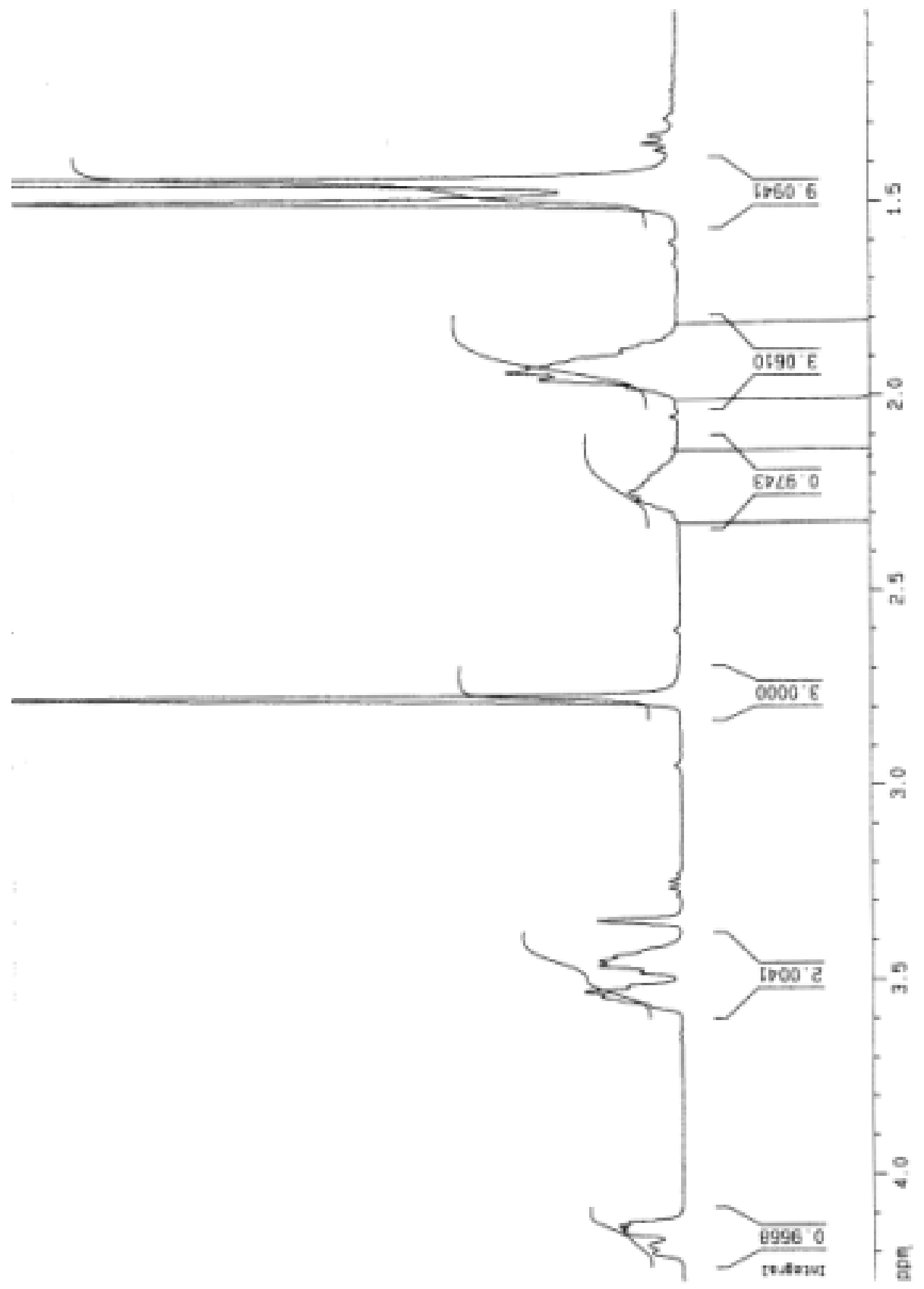




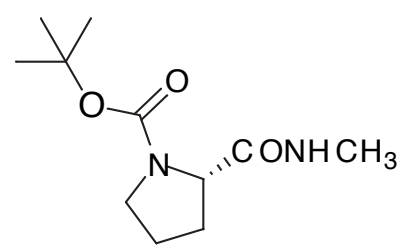

Boc-Pro-NHMe

${ }^{13} \mathrm{C}$ NMR spesctrum at $100 \mathrm{MHz}$ in $\mathrm{CDCl}_{3}$

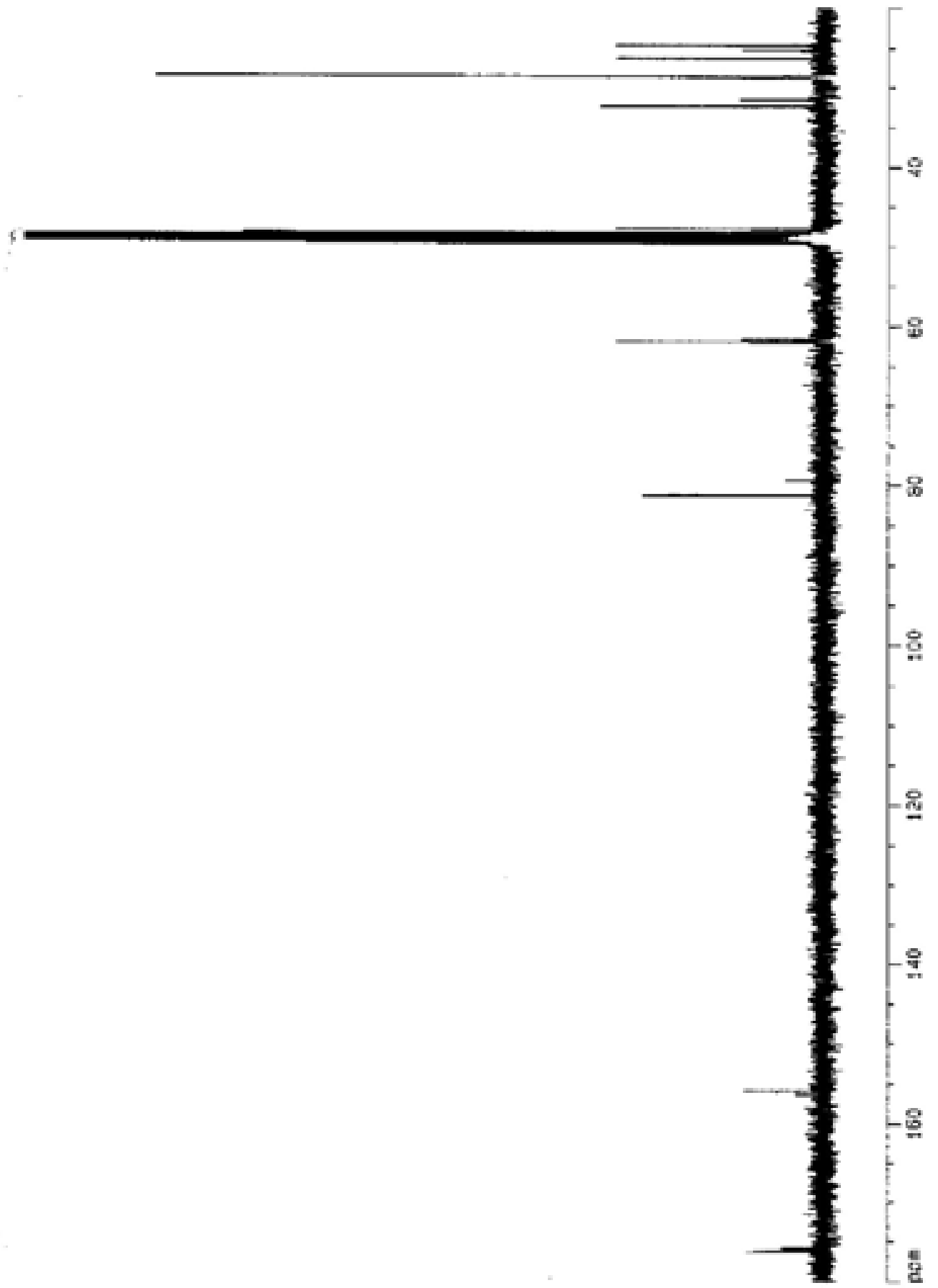




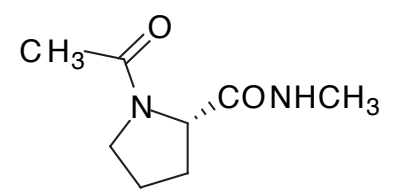

Compound 2

${ }^{1} \mathrm{H}$ NMR spectrum at $400 \mathrm{MHz}$ in $\mathrm{D}_{2} \mathrm{O}$

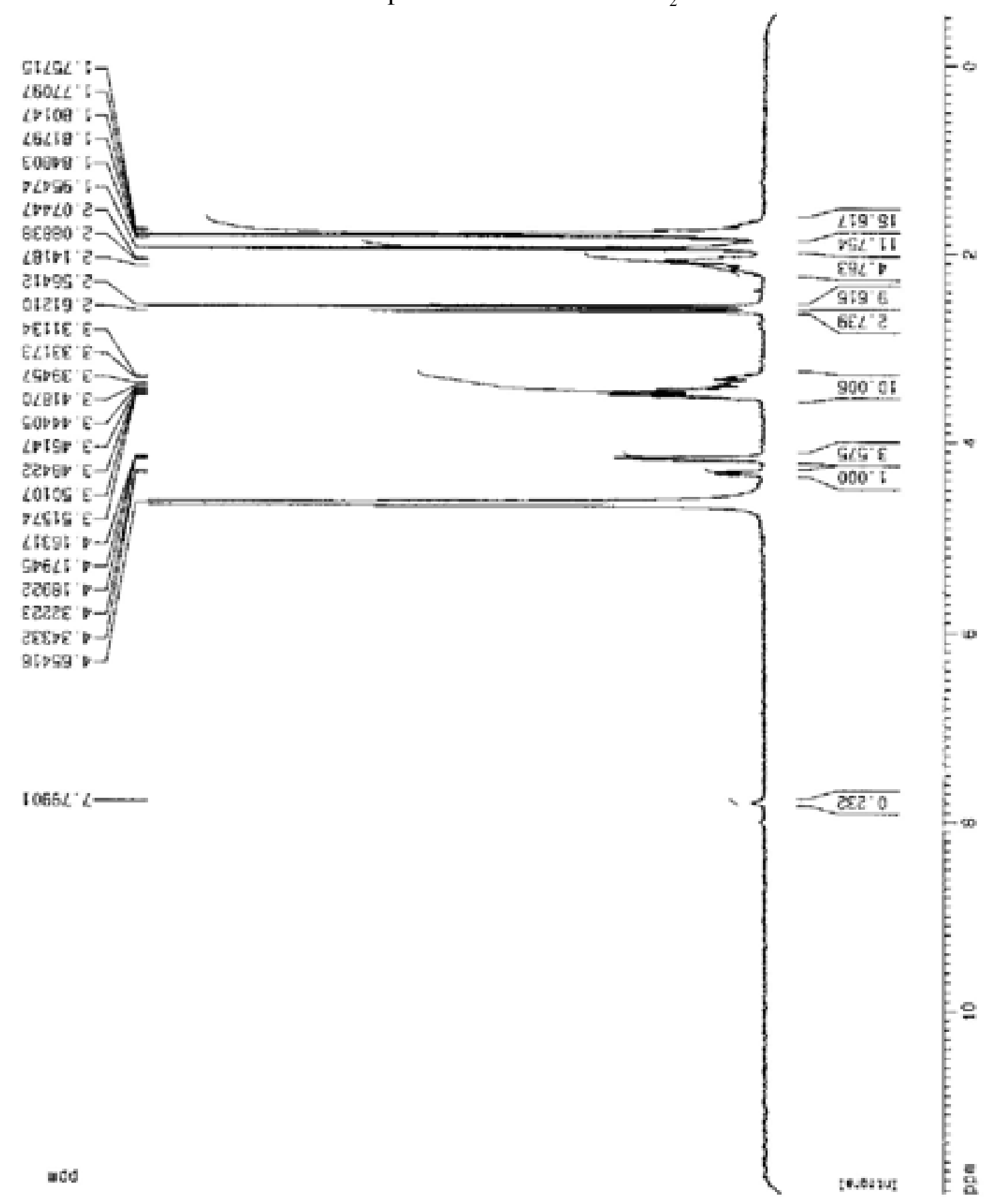




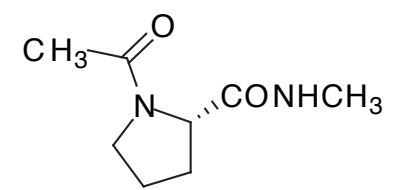

Compound 2

${ }^{13} \mathrm{C}$ NMR spectrum at $100 \mathrm{MHz}$ in $\mathrm{D}_{2} \mathrm{O}$

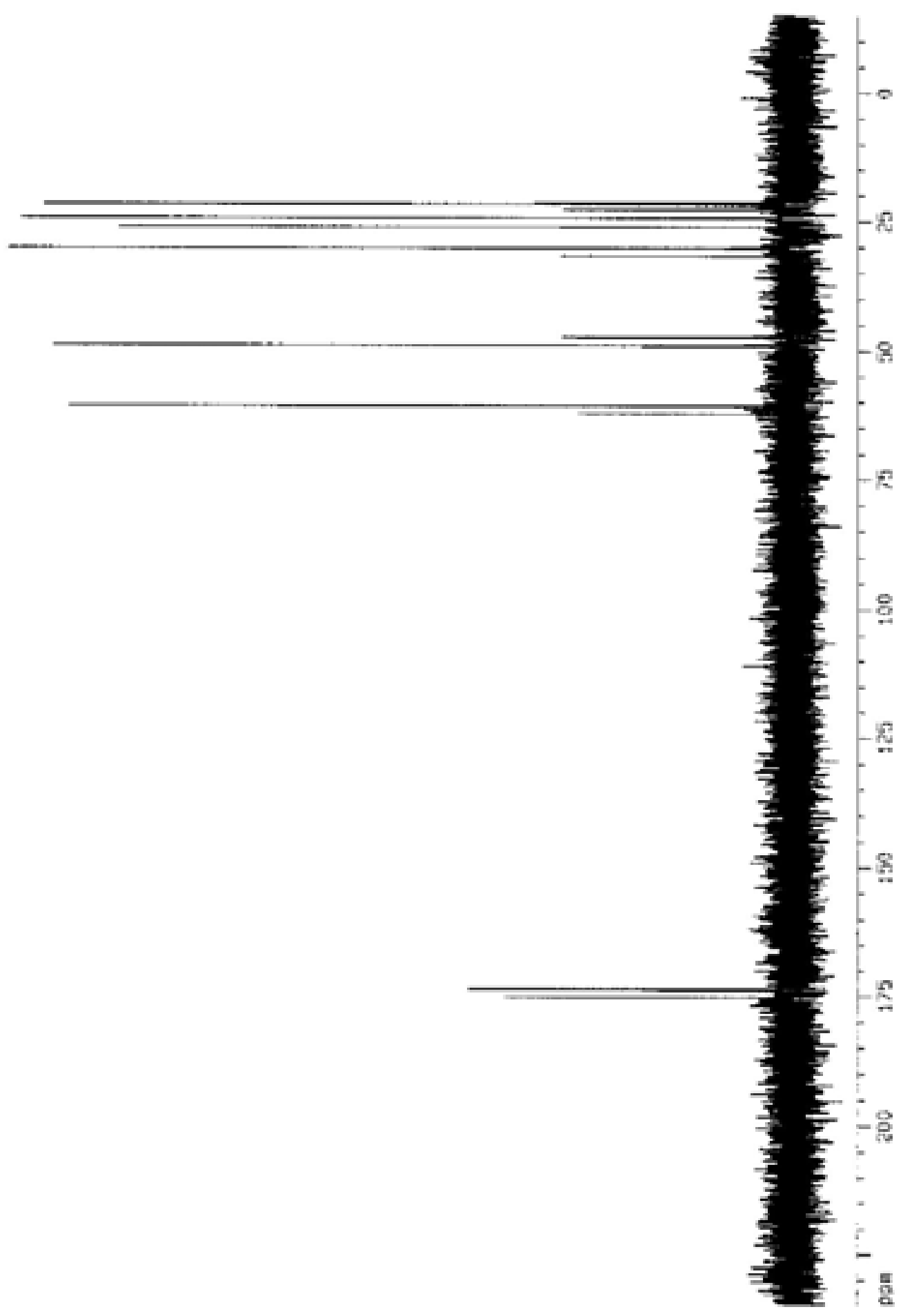


$\mathrm{S} 14$<smiles>CNC(=O)[C@@H]1CCCN1C(=O)CNC(=O)OC(C)(C)C</smiles>

Boc-Gly-Pro-NHMe

${ }^{1} \mathrm{H} \mathrm{NMR}$ spectrum at $400 \mathrm{MHz}$ in $\mathrm{CDCl}_{3}$

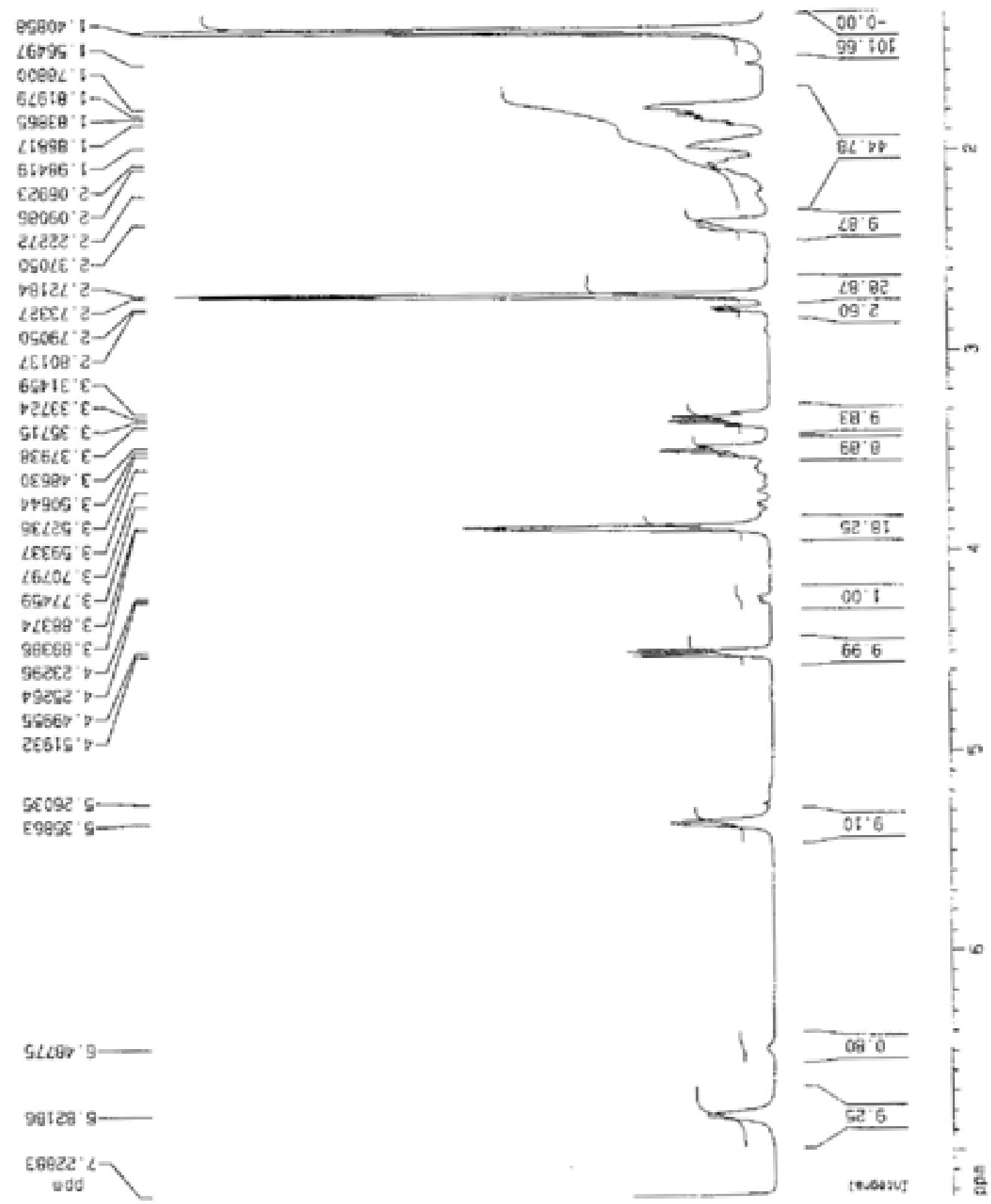


$\mathrm{S} 15$

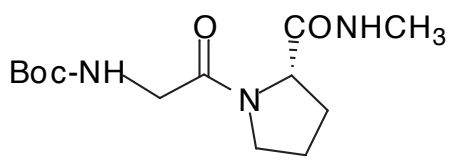

Boc-Gly-Pro-NHMe

${ }^{13} \mathrm{C} \mathrm{NMR}$ spectrum at $100 \mathrm{MHz}$ in $\mathrm{CDCl}_{3}$

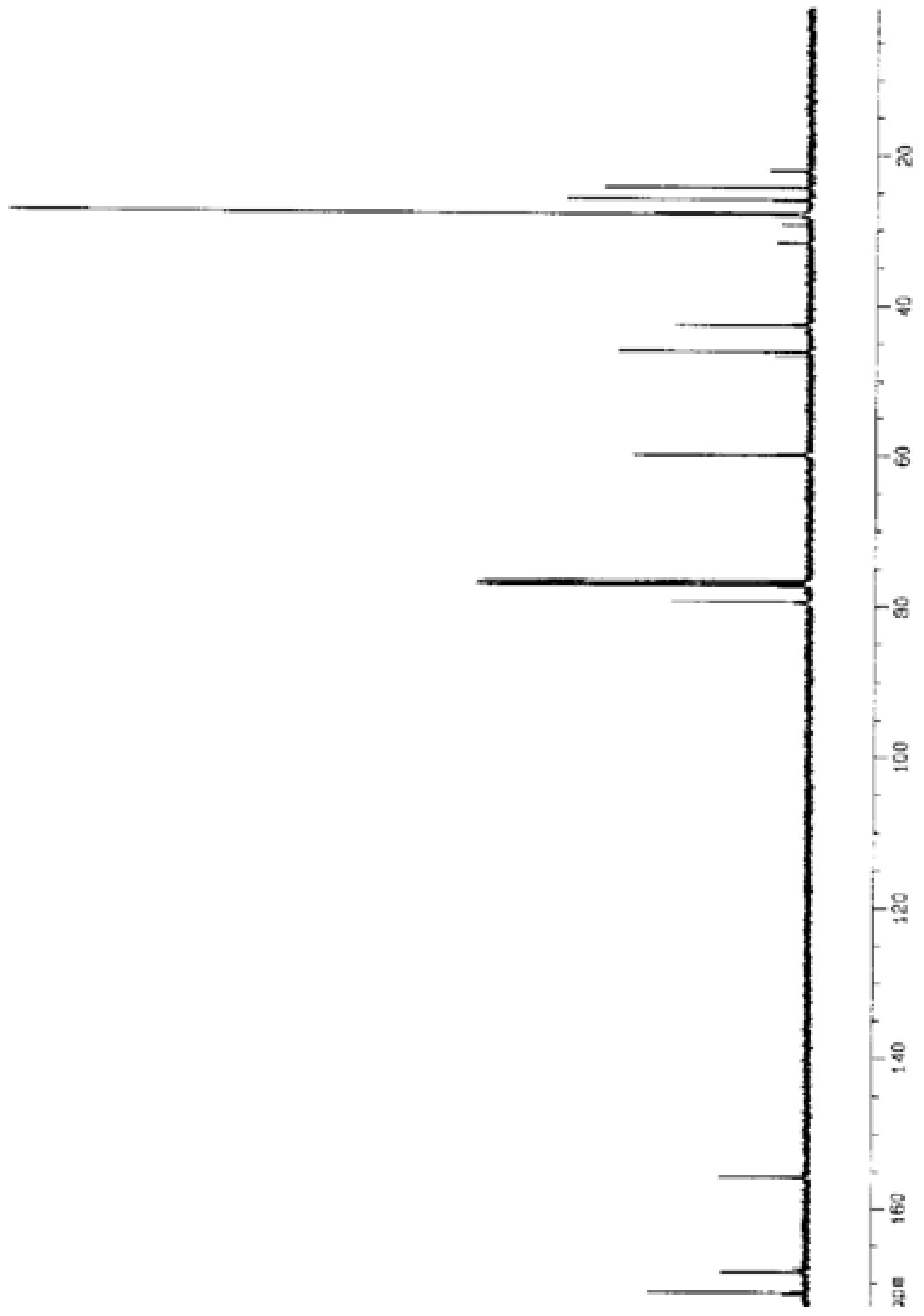


$\mathrm{S} 16$

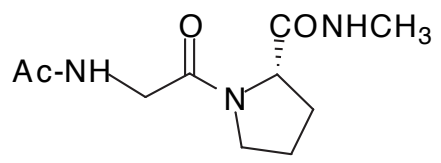

Compound 4

${ }^{1} \mathrm{H}$ NMR spectrum at $400 \mathrm{MHz}$ in $\mathrm{D}_{2} \mathrm{O}$

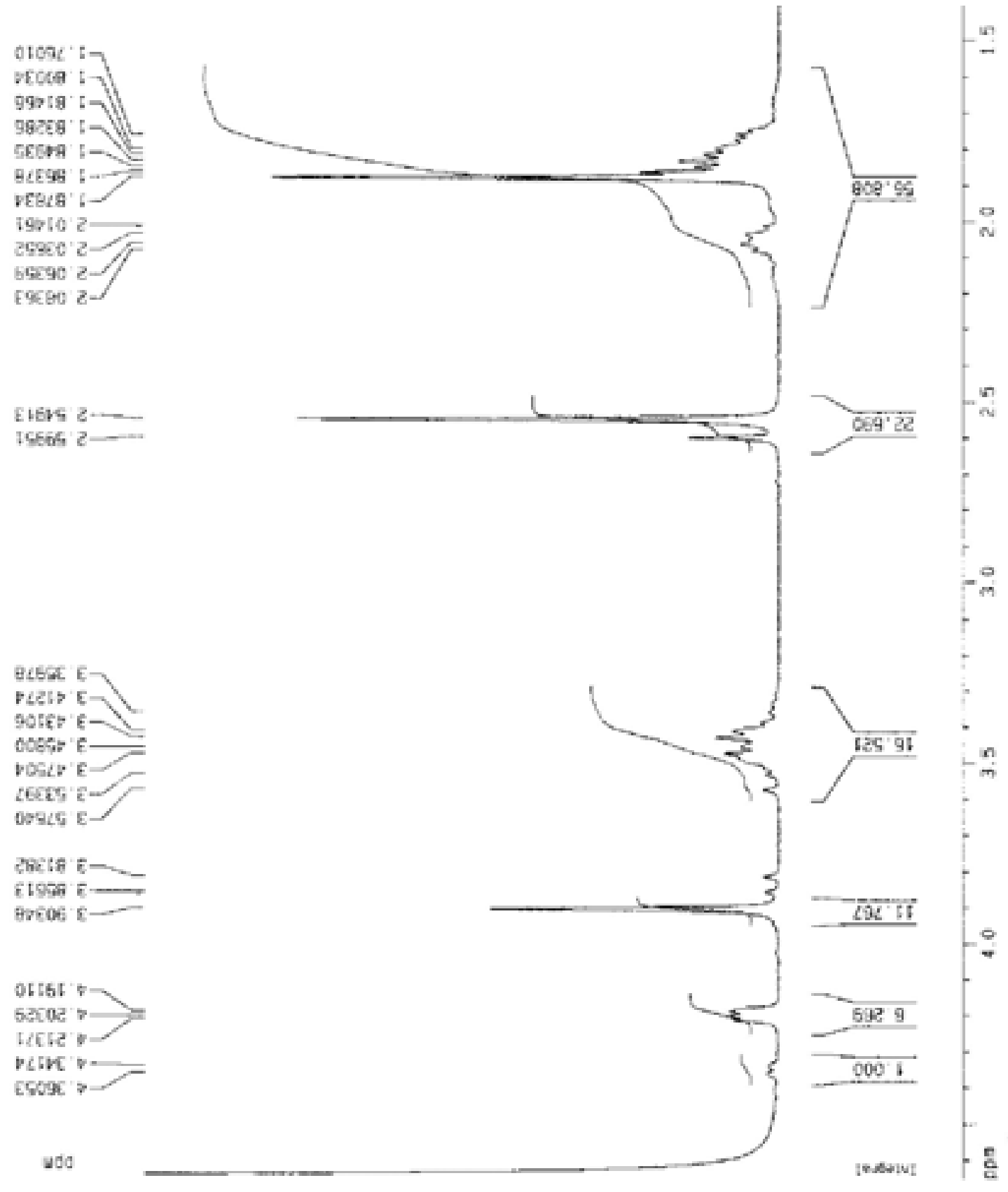


S 17

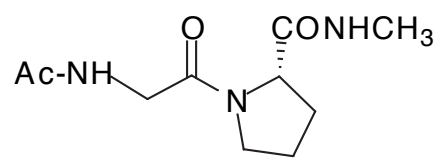

Compound 4

${ }^{13} \mathrm{C}$ NMR spectrum at $100 \mathrm{MHz}$ in $\mathrm{D}_{2} \mathrm{O}$

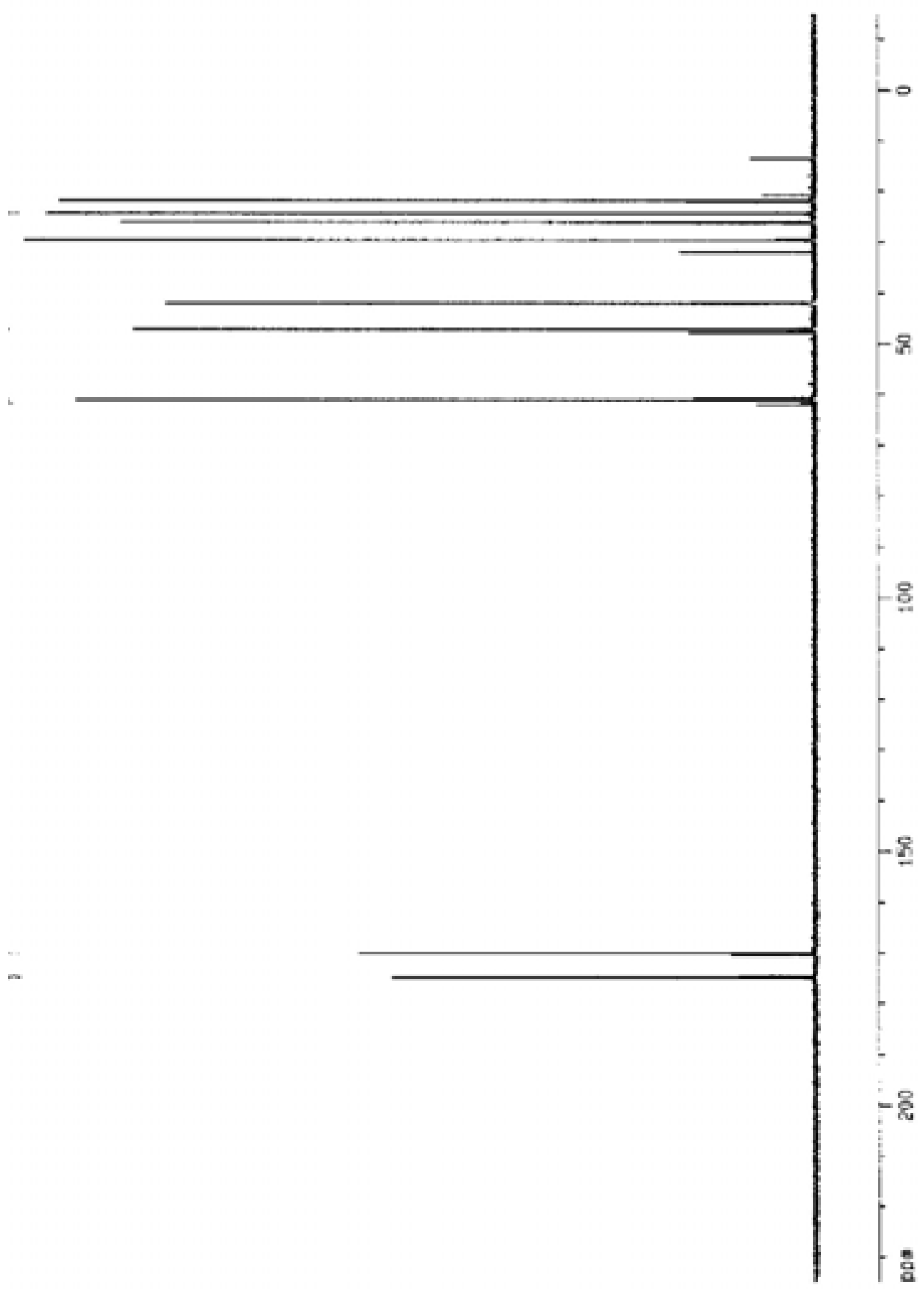


$\mathrm{S} 18$<smiles>CC(=O)N[C@@H](Cc1ccccc1)C(=O)N1CCC[C@H]1C(C)=O</smiles>

Compound 5

${ }^{1} \mathrm{H} \mathrm{NMR}$ at $400 \mathrm{MHz}\left(\mathrm{D}_{2} \mathrm{O}\right)$

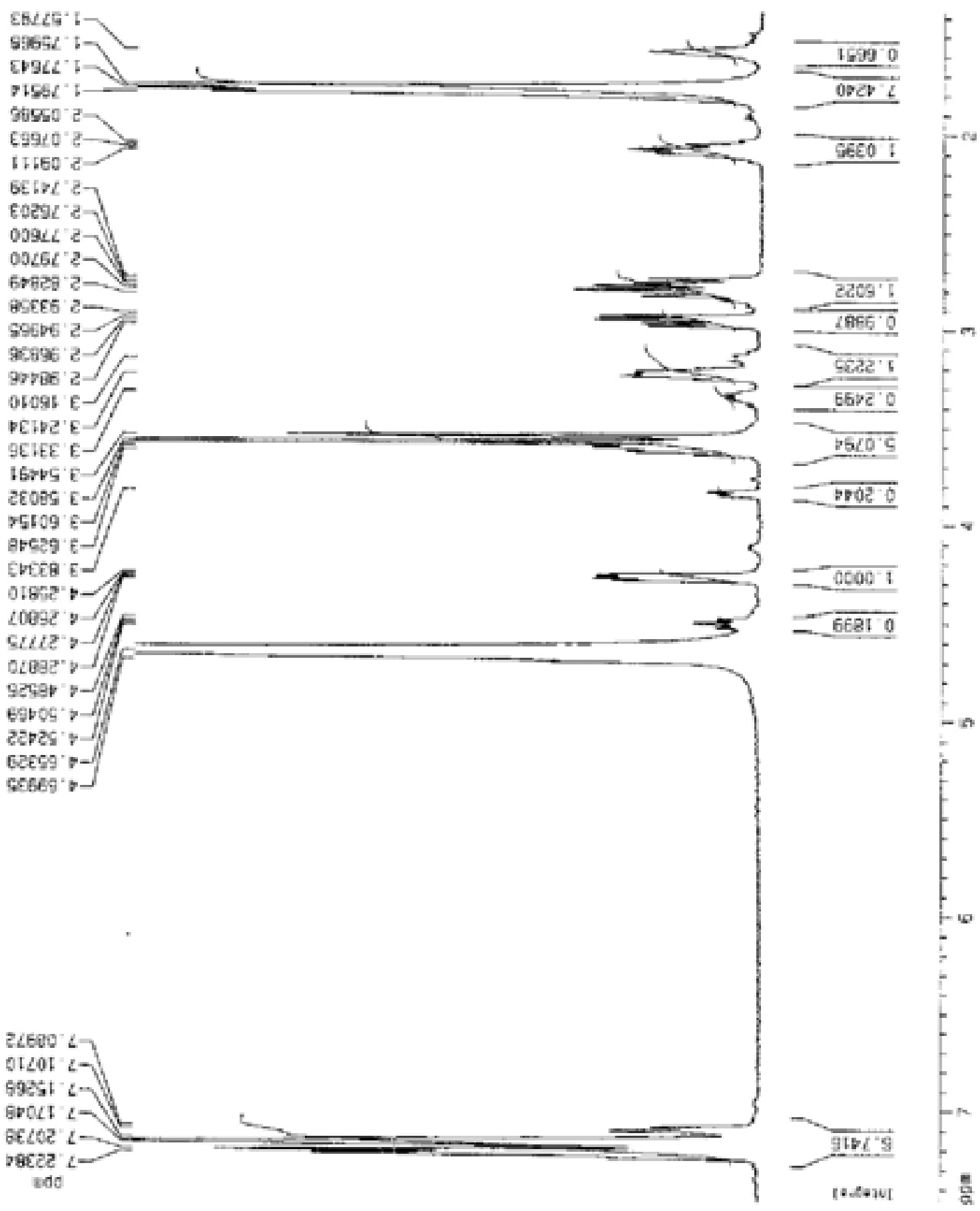




$$
1
$$




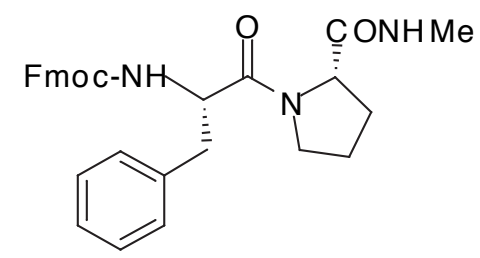

Fmoc-Phe-Pro-NHMe

${ }^{1} \mathrm{H}$ NMR spectrum at $400 \mathrm{MHz}$ in $\mathrm{CDCl}_{3}$

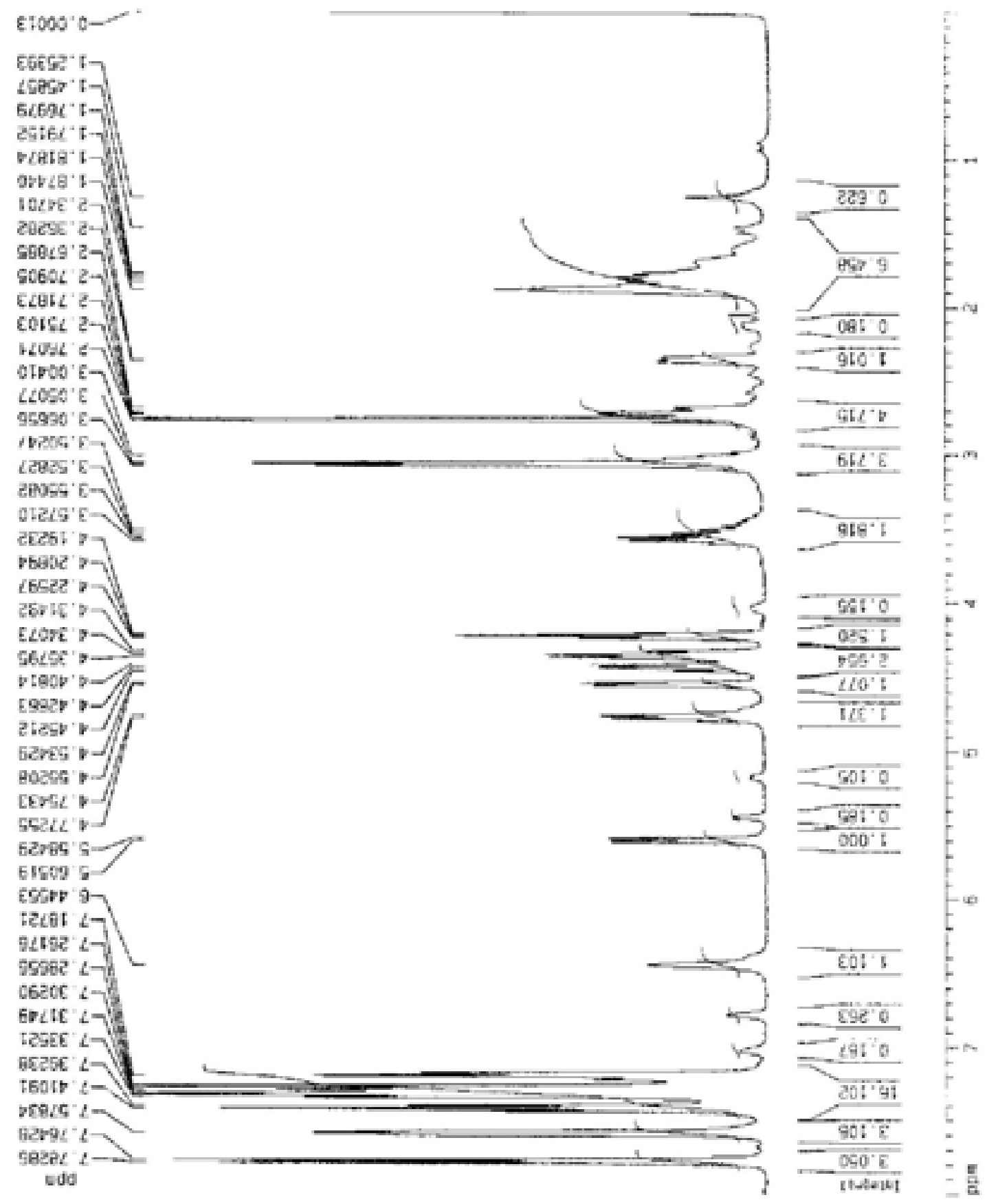




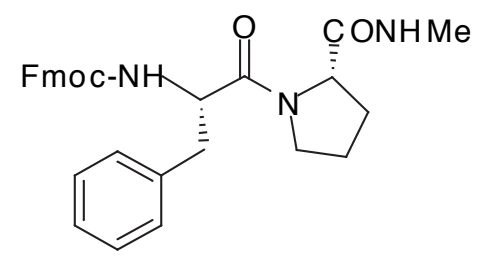

Fmoc-Phe-Pro-NHMe

${ }^{13} \mathrm{C}$ NMR spectrum at $100 \mathrm{MHz}$ in $\mathrm{CDCl}_{3}$

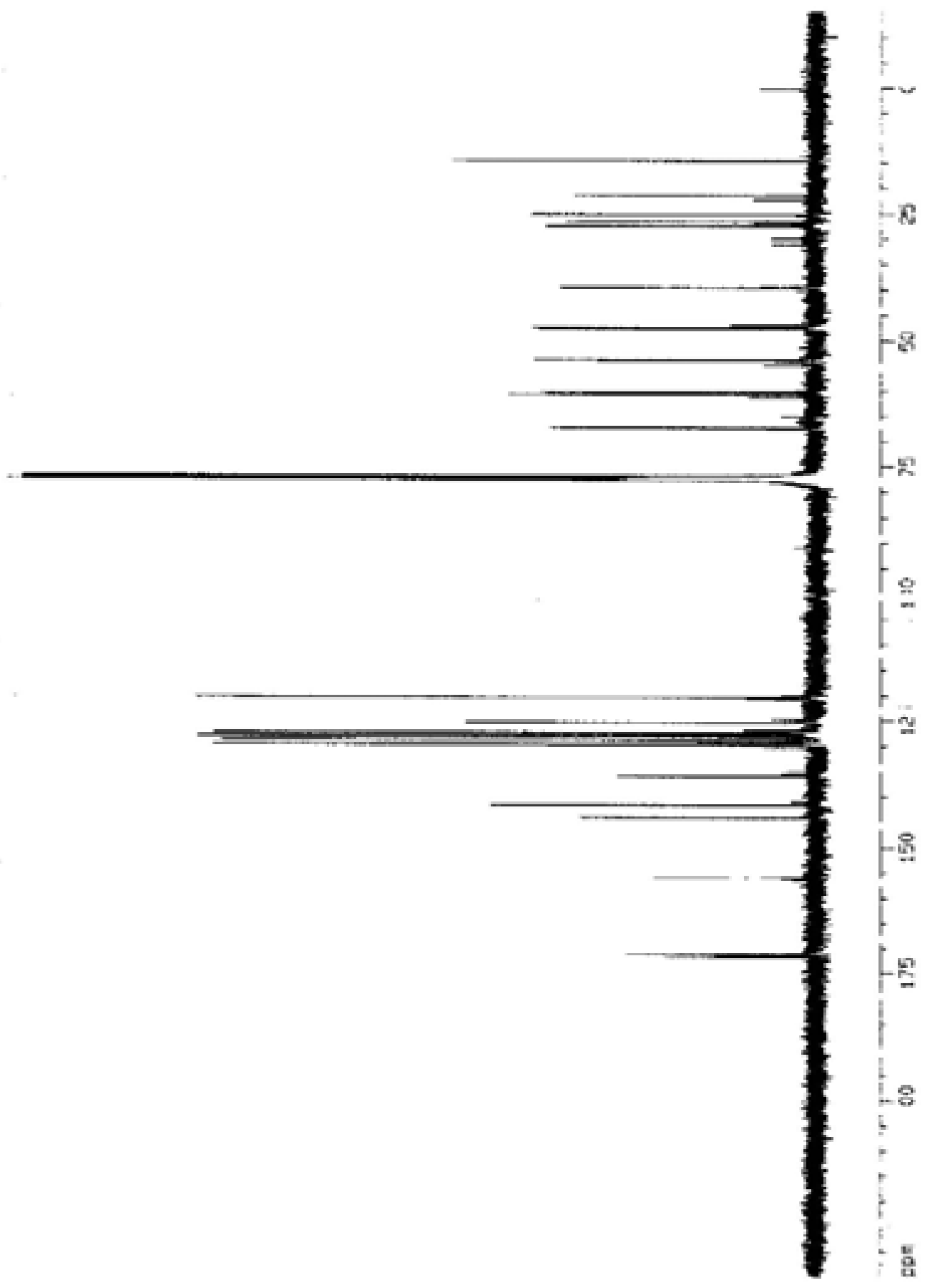




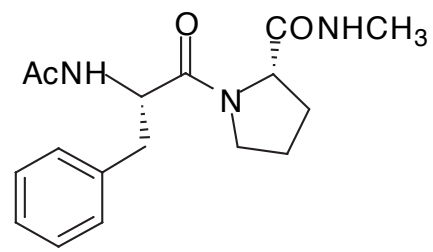

Compound 6

${ }^{1} \mathrm{H} \mathrm{NMR}$ at $400 \mathrm{MHz}$ in $\mathrm{D}_{2} \mathrm{O}$

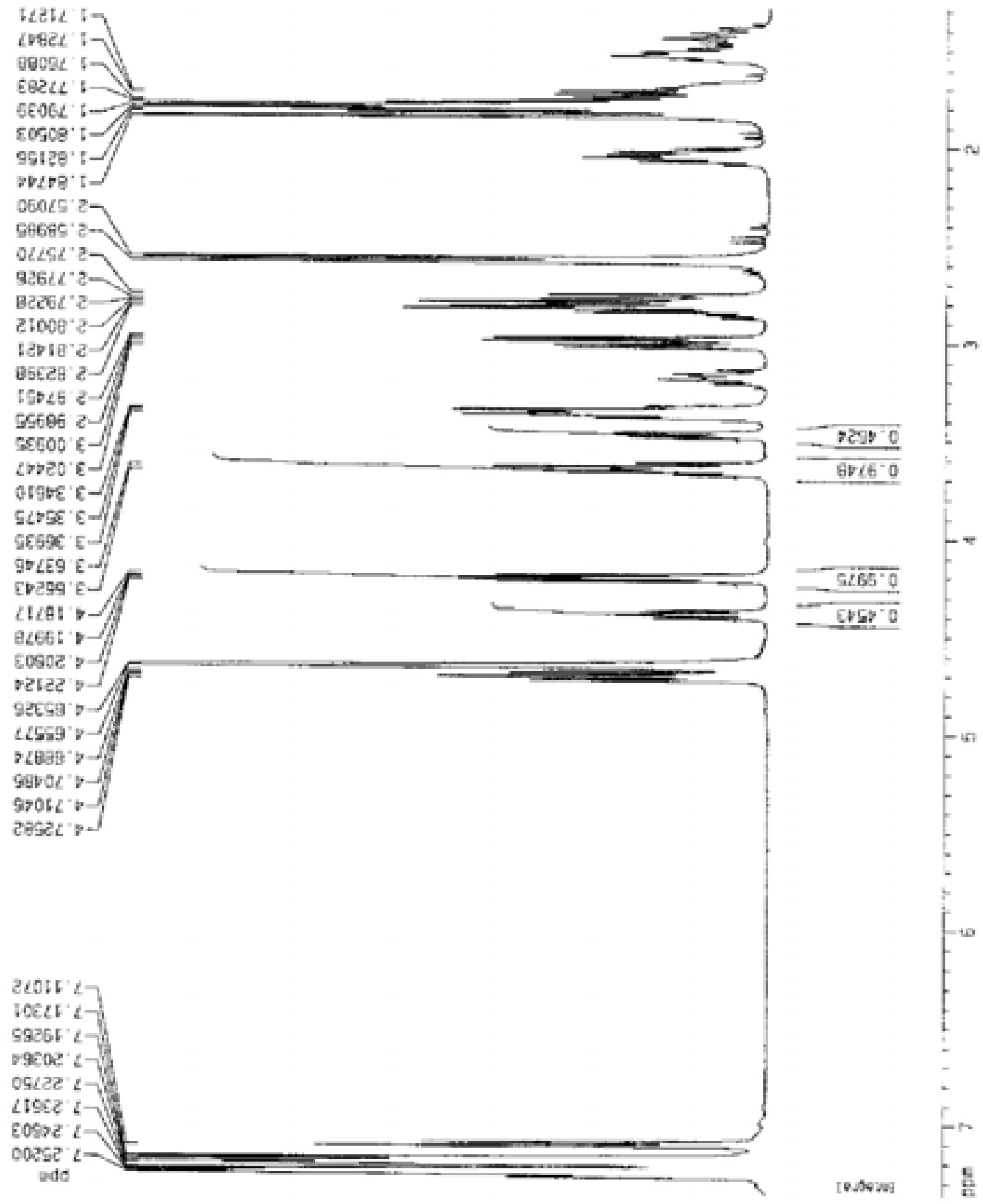


S23

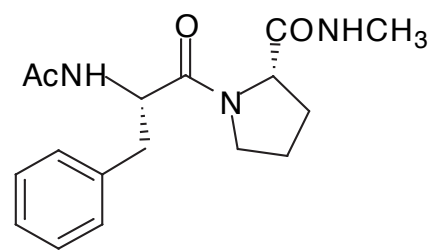

Compound 6

${ }^{13} \mathrm{C}$ NMR at $100 \mathrm{MHz}$ in $\mathrm{D}_{2} \mathrm{O}$

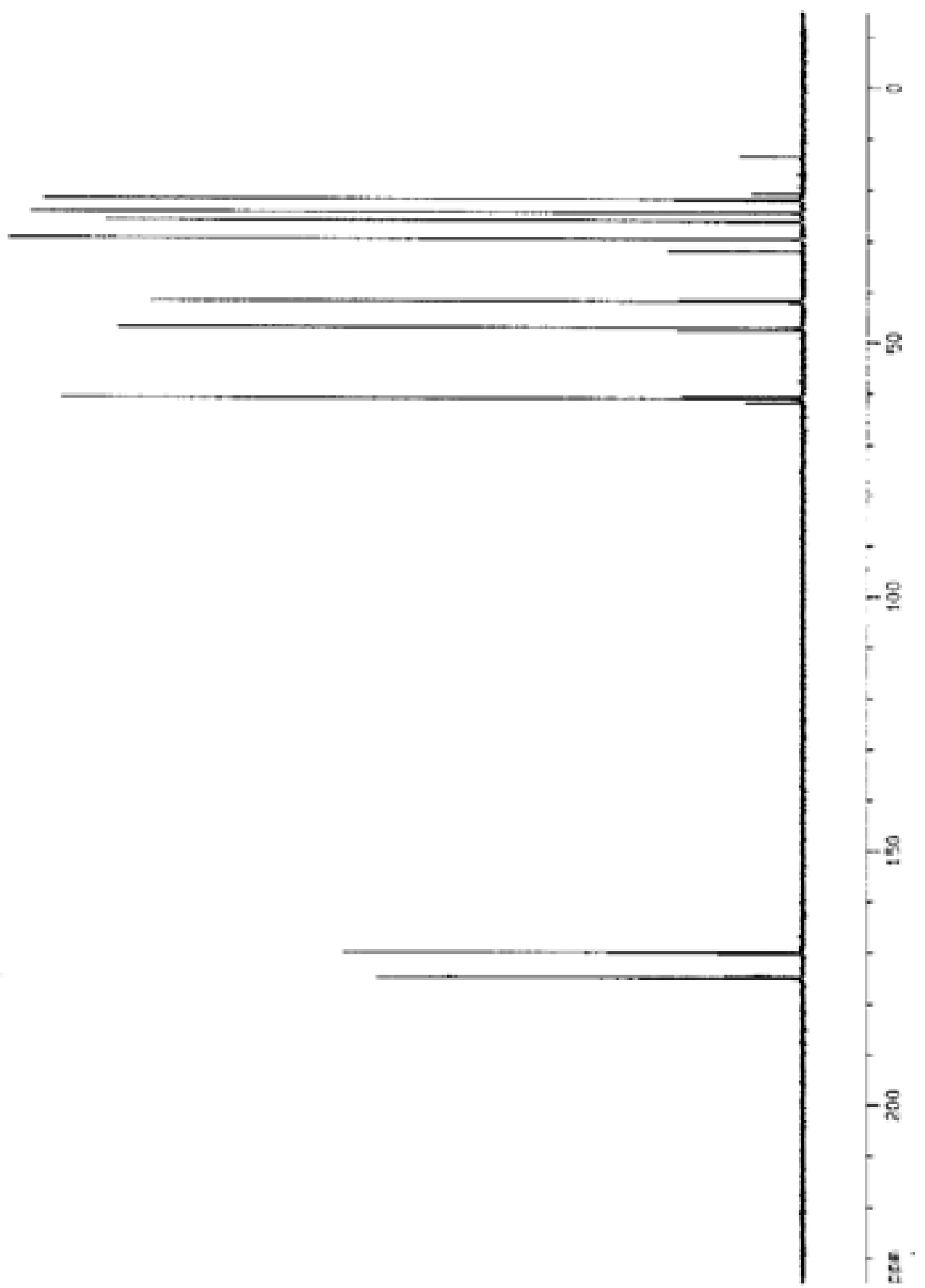


Tables of data from variable temperature NMR experiments

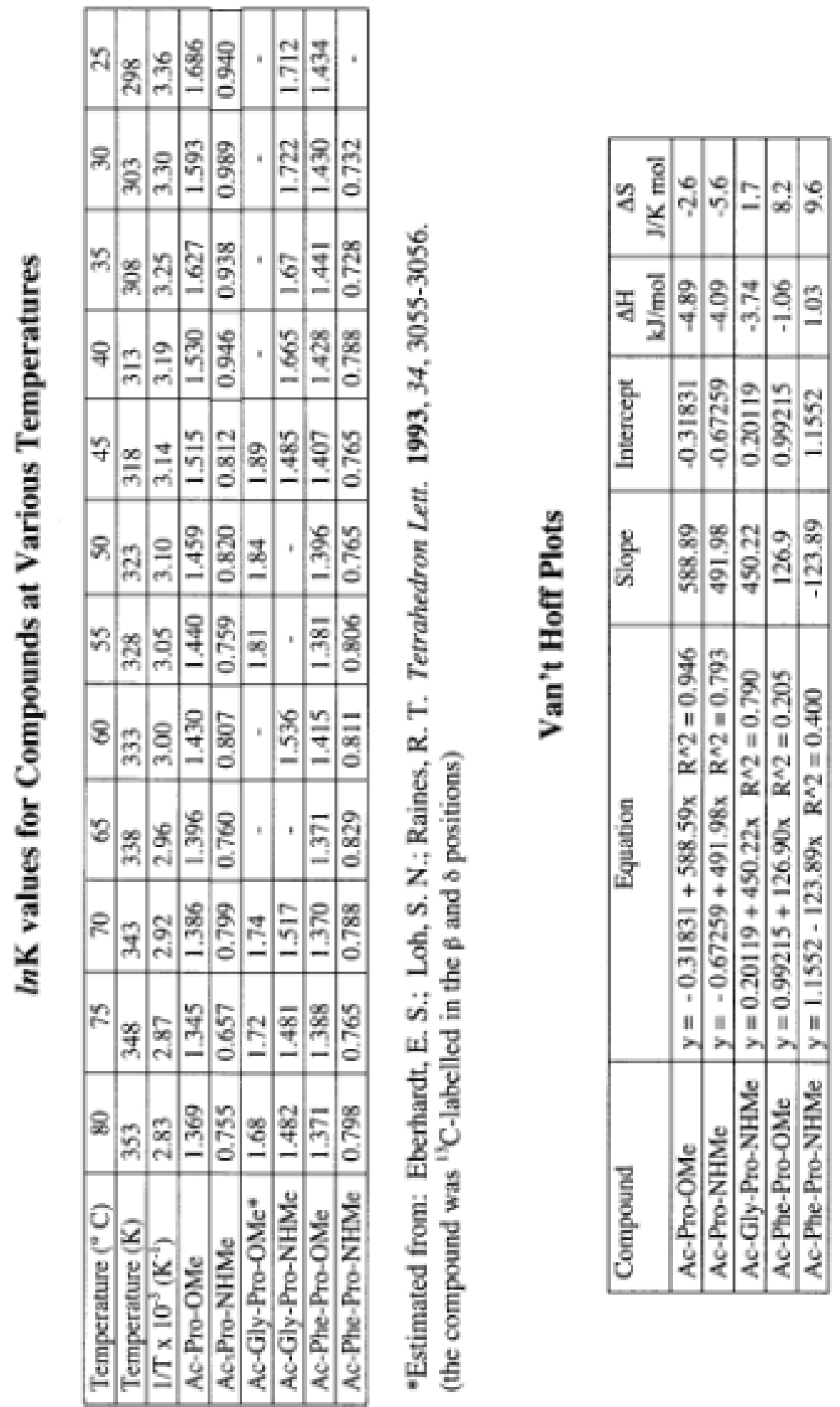




\section{THERMODYNAMIC PARAMETERS FOR COMPOUNDS 1-7.}

\begin{tabular}{lccccc}
\hline Compound & Number & $\begin{array}{c}\mathrm{K}_{t / c} \\
\mathrm{D}_{2} \mathrm{O}, 298 \mathrm{~K}\end{array}$ & $\begin{array}{c}\Delta \mathrm{G}, 298 \mathrm{~K} \\
\left(\mathrm{kcal} \mathrm{mol}^{-1} \mathrm{~K}^{-1}\right)\end{array}$ & $\begin{array}{c}\Delta \mathrm{H} \\
\left(\mathrm{kcal} \mathrm{mol}^{-1}\right)\end{array}$ & $\begin{array}{c}\Delta \mathrm{S} \\
\left(\mathrm{cal} \mathrm{mol}^{-1} \mathrm{~K}^{-1}\right)\end{array}$ \\
\hline Ac-Pro-OMe & $\mathbf{1}$ & $5.40^{a}$ & -0.99 & $-1.17^{b}$ & $-0.62^{c}$ \\
Ac-Pro-NHMe & $\mathbf{2}$ & 2.56 & -0.57 & -0.97 & -1.34 \\
Ac-Gly-Pro-OMe & $\mathbf{3}$ & $\sim 7.2^{d}$ & $-1.20^{d}$ & $-1.27^{d}$ & $-0.25^{d}$ \\
Ac-Gly-Pro-NHMe & $\mathbf{4}$ & 5.54 & -1.01 & -0.89 & +0.41 \\
Ac-Phe-Pro-OMe & $\mathbf{5}$ & 4.2 & -0.83 & -0.25 & +1.96 \\
Ac-Phe-Pro-NHMe & $\mathbf{6}$ & 2.1 & -0.43 & +0.25 & +2.29 \\
Ac-Gly-Phe-Pro-Gly-NH & $\mathbf{7}$ & $4.8^{e}$ & $-0.93^{e}$ & $-0.53^{e}$ & $-1.4^{e}$ \\
\hline
\end{tabular}

${ }^{a}$ Lit. $K_{t / c}=4.6$ in $\mathrm{D}_{2} \mathrm{O}$ at $25{ }^{\circ} \mathrm{C}$ [Bretscher, L. E.; Jenkins, C. L.; Taylor, K. M.; DeRider, M. L.; Raines, R. T. J. Am. Chem. Soc. 2001, 123, 777-778]; ${ }^{b}$ Lit. $\Delta \mathrm{H}=-1.04 \pm 0.02$ kcal mol ${ }^{-1}$ [Eberhardt, E. S.; Panasik, N., Jr.; Raines, R. T. J. Am. Chem. Soc. 1996, 118, 12261-12266]; ${ }^{c}$ Lit $\Delta \mathrm{S}=-0.46 \pm 0.05 \mathrm{cal} \mathrm{mol}^{-1} \mathrm{~K}^{-1}$ [Eberhardt, E. S.; Panasik, N., Jr.; Raines, R. T. J. Am. Chem. Soc. 1996, 118, 12261-12266]; ${ }^{d}$ Results taken from: Eberhardt, E. S.; Loh, S. N.; Raines, R. T. Tetrahedron Lett. 1993, 34, 3055-3056; ${ }^{e}$ Results taken from: Wu, W.-J.; Raleigh, D. P. Biopolymers 1998, 45, 381-394. 